ARTICLE

https://doi.org/10.1038/s41467-018-07763-0

\title{
Chromatin-mediated feed-forward auxin biosynthesis in floral meristem determinacy
}

\author{
Nobutoshi Yamaguchi (10 1,2, Jiangbo Huang ${ }^{3}$, Yoshitaka Tatsumi ${ }^{1}$, Masato Abe ${ }^{1}$, Shigeo S. Sugano (1) ${ }^{2,4}$, \\ Mikiko Kojima $^{5}$, Yumiko Takebayashi ${ }^{5}$, Takatoshi Kiba (1) ${ }^{6}$, Ryusuke Yokoyama ${ }^{7}$, Kazuhiko Nishitani (1) ${ }^{7}$, \\ Hitoshi Sakakibara ${ }^{5,6} \&$ Toshiro Ito ${ }^{1}$
}

In flowering plants, the switch from floral stem cell maintenance to gynoecium (female structure) formation is a critical developmental transition for reproductive success. In Arabidopsis thaliana, AGAMOUS (AG) terminates floral stem cell activities to trigger this transition. Although CRABS CLAW (CRC) is a direct target of $A G$, previous research has not identified any common targets. Here, we identify an auxin synthesis gene, YUCCA4 (YUC4) as a common direct target. Ectopic YUC4 expression partially rescues the indeterminate phenotype and cell wall defects that are caused by the crc mutation. The feed-forward YUC4 activation by $A G$ and $C R C$ directs a precise change in chromatin state for the shift from floral stem cell maintenance to gynoecium formation. We also showed that two auxinrelated direct CRC targets, YUC4 and TORNADO2, cooperatively contribute to the termination of floral stem cell maintenance. This finding provides new insight into the CRC-mediated auxin homeostasis regulation for proper gynoecium formation.

\footnotetext{
${ }^{1}$ Division of Biological Science, Graduate School of Science and Technology, Nara Institute of Science and Technology, 8916-5, Takayama, Ikoma, Nara 6300192, Japan. ${ }^{2}$ Precursory Research for Embryonic Science and Technology, Japan Science and Technology Agency, 4-1-8, Honcho, Kawaguchi-shi, Saitama 332-0012, Japan. ${ }^{3}$ Temasek Life Sciences Laboratory, 1 Research Link, National University of Singapore, Singapore 117604, Republic of Singapore. ${ }^{4}$ Ritsumeikan Global Innovation Research Organization, Ritsumeikan University, 1-1-1, Shiga 525-8577, Japan. ${ }^{5}$ RIKEN Center for Sustainable Resource Science, 1-7-22, Suehiro, Tsurumi, Yokohama 230-0045, Japan. ${ }^{6}$ Department of Applied Biosciences, Graduate School of Bioagricultural Sciences, Nagoya University, Chikusa, Nagoya 464-8601, Japan. ${ }^{7}$ Graduate School of Life Sciences, Tohoku University, Aoba-ku, Sendai 980-8578, Japan. Correspondence and requests for materials should be addressed to T.I. (email: itot@bs.naist.jp)
} 
F lowers are reproductive organs in angiosperms ${ }^{1}$. Each flower is composed of four different types of floral organs: sepals, petals, stamens, and carpel ${ }^{1}$. These floral organs are generated from floral meristems, which each contain a small pool of pluripotent and undifferentiated stem cells ${ }^{2}$. To form the appropriate number of floral organs, the rate of stem cell proliferation must be coordinated with the rate of organ formation. Such balances are determined by floral meristem termination (also known as floral determinacy). If floral meristems terminate too early, the cells are unable to produce the precise number of floral organs. In contrast, delayed or no floral meristem termination leads to failure in reproductive organ and sterility. Thus, floral meristem termination is critical for allocating resources for the next generation.

The MADS-box transcription factor AGAMOUS (AG) is considered to be a master regulator of floral meristem termination in Arabidopsis $^{3}$. A loss of AG function results in indeterminate growth of floral meristems ${ }^{3-5}$. Interplay between AG and APETALA2 (AP2) regulates the expression of hundreds of targets genes ${ }^{6}$. Previous studies have only identified a handful of direct AG targets that control determinacy. Such targets include the homeodomain transcription factor WUSCHEL (WUS) $^{7,8}$, the C2H2 zinc-finger protein KNUCKLES (KNU) $)^{9-11}$, and the YABBY transcription factor CRABS CLAW (CRC) ${ }^{12-17}$. WUS has a central role in floral meristem determinacy. AG binds directly to nucleotide sequences near the WUS transcription start site and in its $3^{\prime}$ UTR to repress its expression ${ }^{8}$. AG also binds to the promoter of a WUS repressor, $K N U$, at the site where a CArG box and a Polycomb response element (PRE) overlap ${ }^{10,11}$. Polycomb group (PcG) proteins bind to the PRE to deposit/maintain histone 3 lysine 27 (H3K27) trimethylation to silence transcription ${ }^{18,19}$. Eviction of the PcG proteins by AG triggers $K N U$ expression in a cell division-dependent manner and hence suppresses WUS expression ${ }^{11}$. CRC expression is also directly controlled by $A G^{14,15,17}$. CRC represses TORNADO2 (TRN2) via an evolutionarily conserved and biologically functional cis-regulatory motif ${ }^{17,20,21}$. TRN2 modulates homeostasis of the morphogenic plant hormone auxin and contributes to WUS regulation ${ }^{17}$. Previous genetic evidence strongly suggests that an auxin-related target(s) acts downstream of CRC and in parallel with TRN2 ${ }^{17}$. Auxin transport inhibitor treatment led to an almost full rescue of the $c r c$ mutant phenotype, while a trn 2 mutation led to a partial rescue $^{17,22}$. Auxin homeostasis is controlled by multiple key regulators. For example, the two-step conversion of tryptophan to indole-3-acetic acid catalyzed by TRYPTOPHAN AMINOTRANSFERASE OF ARABIDOPSISs (TAAs) and YUCCAs (YUCs) is a critical step in the major pathway synthesizing bioactive auxin ${ }^{23-25}$. Auxin perception and signaling are controlled by auxin receptors, ubiquitin-ligase, and auxin-dependent transcriptional modules ${ }^{26}$. Despite their potential impact on auxin homeostasis, auxin-related CRC targets, other than TRN2, have not been identified.

After termination of floral meristems, rapid cell expansion and elongation direct organ growth and development. Auxin maxima in organ primordia correlate well with the maximal rates of primary cell wall expansion, which mainly requires cellulose, non-cellulosic polysaccharides, pectin, and glycoproteins $\mathrm{s}^{27-30}$. Auxin results in the loosening and expansion of cell walls ${ }^{31}$, which requires interactions between multiple molecular factors to relax wall tension. Expansins are major regulators that mediate the local sliding of wall polymers by reducing adhesion between adjacent wall polysaccharides ${ }^{32}$. Xyloglucan endotransglucosylases/hydrolases catalyze cleavage of xyloglucan polymers and reconnect xyloglucan chains ${ }^{33}$. Although local differences in cell wall composition have an important role in meristem activity and its subsequent development, little is known about cell wall composition during floral meristem termination and their effects on stem cell activities.
In this study, we systematically identified genes that are transcriptionally regulated by both AG and CRC. We show that the auxin biosynthetic gene YUC4 is a common direct target for both $A G$ and CRC. AG and CRC synergistically regulate YUC4 expression during floral meristem determinacy. Furthermore, we showed that two auxin-related CRC targets, YUC4 and TRN2 cooperatively function for floral meristem termination. Our results provide insight into the transcriptional cascades that govern the transition from floral stem cell maintenance to gynoecium formation.

\section{Results}

AG and CRC control cellular developmental processes. To identify target genes that are regulated by both AG and CRC, we computationally reanalyzed four published transcriptome datasets $s^{6,17,34}$. First, we screened for genes that were differentially expressed in both $c r c-1 \quad k n u-1$ vs $k n u-1$, and ag-12 vs WT (Fig. 1a). This analysis identified 332 upregulated and 686 downregulated genes. Then, we examined how many of these genes were differentially expressed in opposite directions in the two ap 2 mutants ${ }^{6}$. Among the 1018 genes, 53 genes were restored to wild-type levels by introducing ap2 mutations (Fig. 1a, b, Supplementary Fig. 1, and Supplementary Data 1).

To examine the potential functions of the 53 putative AG and CRC regulated genes, we performed a GO term enrichment analysis using agriGO and REVIGO ${ }^{35}$ (Supplementary Fig. 2 and Supplementary Data 2). Three major categories were visualized with an interactive graph view and TreeMap (Fig. 1c, d): Regulation of cellular processes, Cellular developmental processes, and Responses to organic substances. Regulation of cellular processes included GO terms related to transcription, such as 'gene expression', 'RNA metabolism', and 'RNA biosynthesis' (Fig. 1c, d). A cellular developmental process included the GO terms 'cellular developmental process', 'anatomical structure morphology', 'anatomical structure development', and 'shoot system development' (Fig. 1c, d). The GO term 'anatomical structure process' was associated with YUC4, and EXPANSIN 8 (EXP8), which encoded an enzyme in the auxin biosynthesis pathway ${ }^{25}$ and an enzyme that alters cell wall polymers $^{36}$, respectively. The Responses to organic substances category included various responses such as 'response to chemical', 'cellular response to stimulus', 'response to stress', 'response to endogenous stimulus', and 'cellular response to stimulus' (Fig. 1c, d). The genes associated with this category included ARABISOPSIS THALIANA CELL WALL INVERTASE1 $(\text { AtcwINV1 })^{37}$, BR-ENHANCED EXPRESSION (BEE2) ${ }^{38}$, and ARABIDOPSIS RESPONSE REGULATOR15 (ARR15) ${ }^{39}$.

Then, we investigated how many of these 53 genes were bound by AG using a publicly available AG ChIP-seq dataset ${ }^{40}$. Among the 53 genes, 9 genes were significantly enriched by AG (Fig. 1b, red square). The 9 genes regulated by both AG and CRC were MITOGEN-ACTIVATED PROTEIN KINASE 1 (At1g10210) ${ }^{41}$, ARABIDOPSIS THALIANA HOMEOBOX PROTEIN 2 $(A T H B 2)^{42}$, wound-responsive family protein (At4g28240), HALF FILLED $(H A F)^{43}$, SHATTERPROOF $2(S H P 2)^{44}$, Sec14p-like phosphatidylinositol transfer family protein (At1g05370), TARGET OF EAT $3(\text { TOE } 3)^{45}$, GLABROUS INFLORESCENCE STEMS $(G I S)^{46}$, and $Y U C 4^{25}$. Since a previous study proposed the existence of an auxin-related target (or targets) that act downstream of CRC and in parallel with TRN $2^{17}$, we hypothesized that YUC4 could be such a target.

AG and CRC synergistically regulate YUC4 expression. We performed a quantitative reverse transcription-polymerase chain reaction (qRT-PCR) analysis to confirm the microarray results using total RNA extracted from floral bud clusters up to stage 7 . 
a

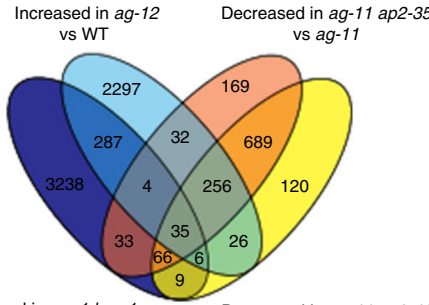
vs knu-1

Decreased in ag-11 ap2-43 vs ag-11

C
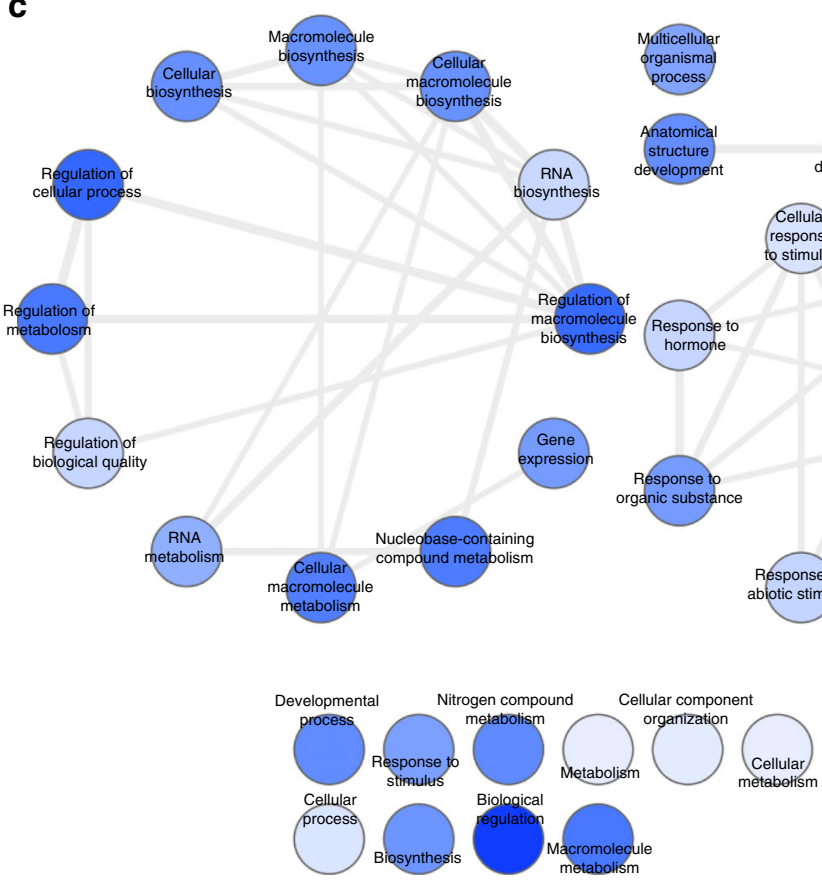

Primary

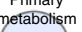

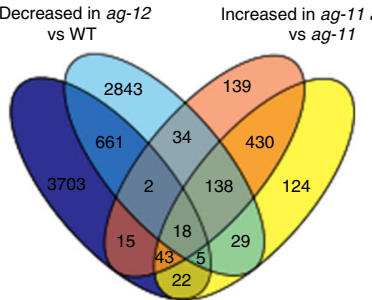

Increased in ag-11 ap2-43 vs ag-11 b

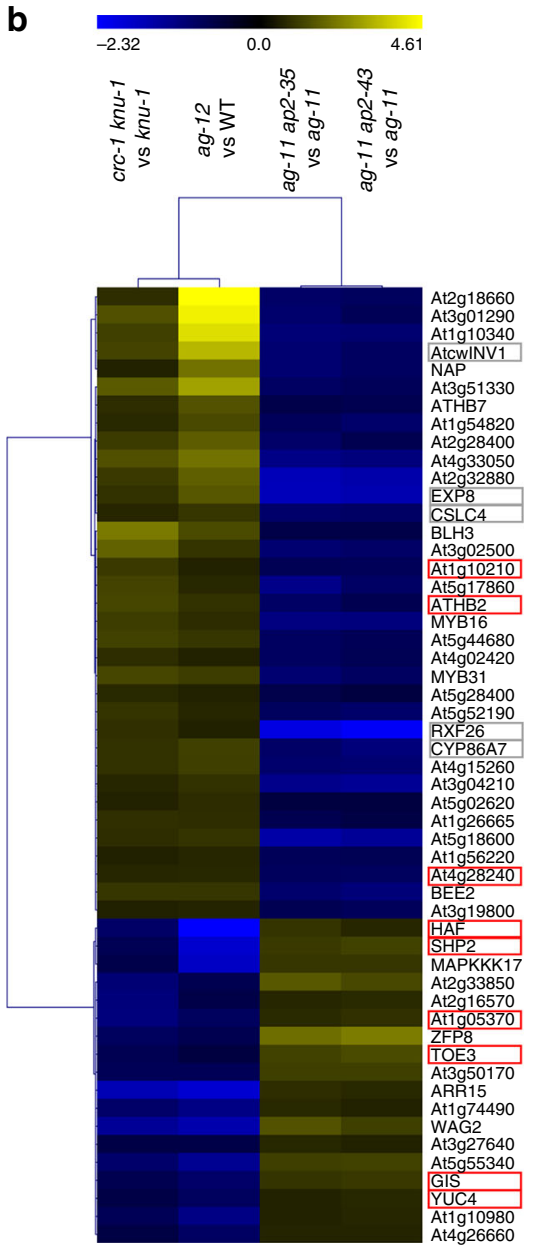

\section{d}

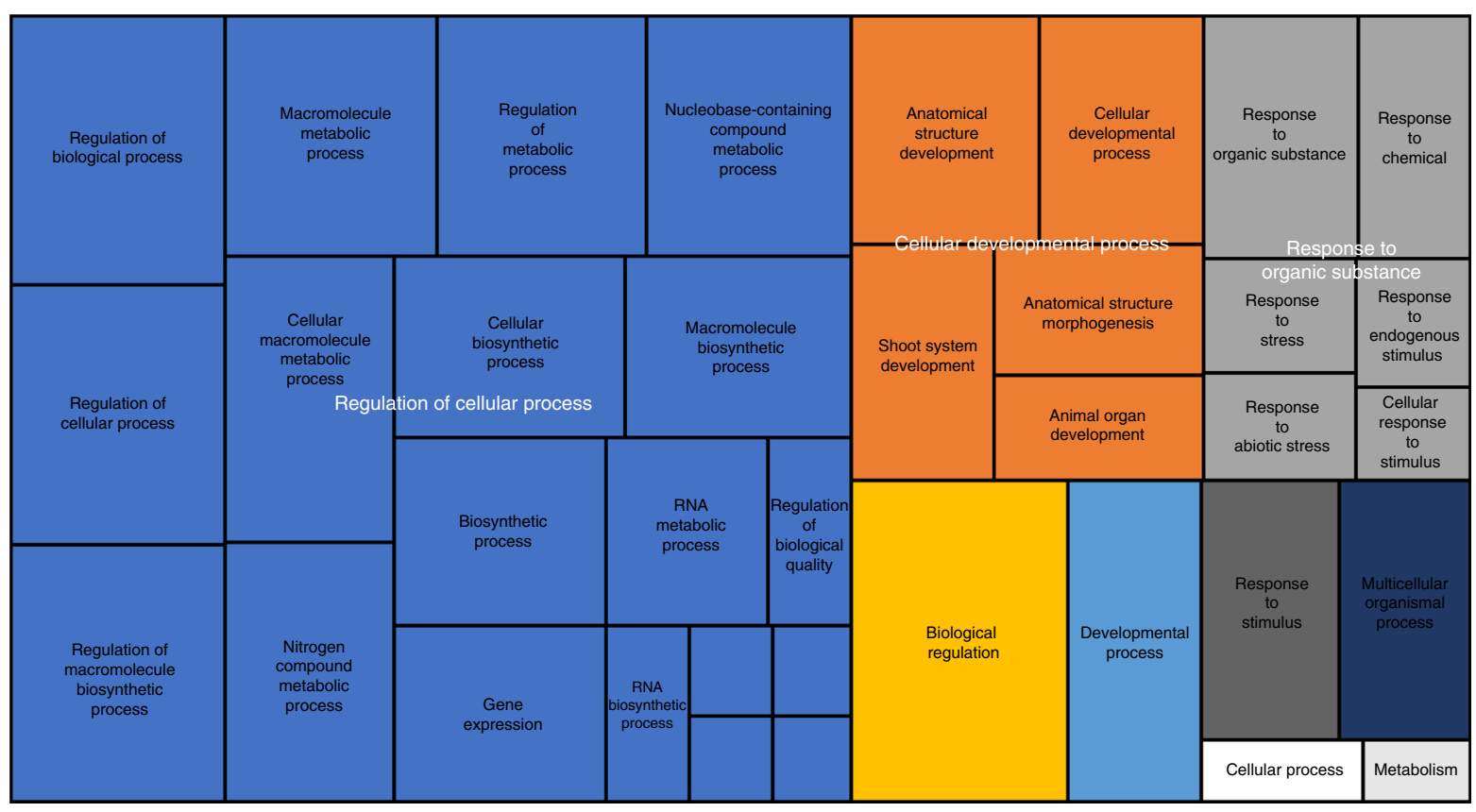

Fig. 1 Identification of downstream targets of AG and CRC during flower development. a Venn diagrams showing the overlap between genes with increased/decreased expression in crc-1 knu-1 vs knu-1 and ag-12 vs WT, and genes with decreased/increased expression in ag-11 ap2-35 vs ag-11 and ag- 11 ap2-43 vs ag-11. $\mathbf{b}$ Hierarchical clustering of 53 genes downstream of AG and CRC. The heat map displays the $\log _{2}$ fold change of differentially expressed genes based on public datasets. Red and gray boxes highlight AG direct targets and cell wall regulators, respectively. c Interactive graph view generated by REVIGO. Dark and light colors indicate lower and higher $p$-values, respectively. Circle sizes indicate the frequency of the GO term in the underlying GOA database. d TreeMap view of GO terms 
Similar to previous microarray data, we also detected a reduction in YUC4 transcript levels in the ag-1 mutant compared with the wild type (Fig. 2a). YUC4 mRNA levels were also lower in crc knu than in knu (Fig. 2b). Low levels of YUC4 expression were also found in the $\mathrm{crc}$ single mutant (Fig. 2c). A further reduction in YUC4 transcript levels was observed in the ag-1-/+ $c r c-1$ mutant (Fig. 2c), which was accompanied by a reduction in floral meristem determinacy, when compared to the $\mathrm{crc}$ single mutant as previously reported ${ }^{12}$ (Supplementary Fig. 3). In addition, the expression of the stem cell determinant, WUS, increased in the $a g-1-/+c r c-1$ mutant relative to the $c r c-1$ mutant (Supplementary Fig. 3).

To investigate YUC4 expression, we generated a $p Y U C 4:: G U S$ line. The $3.7 \mathrm{~kb}$ upstream of the YUC4 promoter recapitulated the YUC4 mRNA distribution pattern: highest signals were detected in the abaxial side of epidermal cells in carpels at stage 6 in both the independent T1 YUC4 reporter lines and by YUC4 mRNA in situ hybridization (Supplementary Fig. 4). At stage 7, YUC4 expression was restricted to the tip of carpels (Supplementary Fig. 4). This expression pattern is similar to that reported previously ${ }^{47}$. A spatial expression test revealed that the expression domains of the CRC mRNA, CRC protein, and YUC4 reporter overlapped in the abaxial carpels at stage 6 of flower development in the wild type (Fig. 2d and Supplementary Fig. 5). Although we detected the effects of $a g$ or $\operatorname{crc}$ mutations on YUC4 expression using floral bud clusters up to stage 7 by RT-PCR, the observed expression patterns could be due to secondary effects such as tissue composition differences. We used two different approaches to confirm the contribution of AG and CRC to YUC4 transcription. In the first approach, we examined the spatial expression patterns of YUC4 in the mutant background at stage 6 , where tissue composition differences are minimal. In the $a g-1$ mutant, YUC4 was mostly limited to the presumptive nectaries at the base of the third whorl (Fig. 2e). While the YUC4 signal intensity and expression pattern was similar between $k n u-1$ and the wild type, YUC4 expression in the crc-1 knu-1 and crc-1 mutants was reduced specifically in the abaxial carpels at stage 6 of flower development in the wild type (Fig. $2 \mathrm{f}-\mathrm{h}$ ). Reduction of auxin synthesis genes in $\mathrm{crc}-1$ could be dependent on YUC4 since TAA1 expression was not affected in $c r c-1$ as in the wild type ${ }^{24}$ (Supplementary Fig. 6). Furthermore, YUC4 expression was lower in $a g-1-1+c r c-1$ than in the wild type (Fig. 2i). Although YUC4 is expressed in sepals, no difference in YUC4 expression was observed in any of the mutants tested here, suggesting that YUC4 expression in sepals is not regulated by AG or CRC.

In the second approach used to examine the roles of $A G$ and CRC in regulating YUC4 expression, we monitored YUC4 expression after a short induction of AG and/or CRC. We expressed AG or CRC proteins fused to a glucocorticoid receptor hormone binding domain (GR). Nuclear entry of the AG-GR fusion protein by dexamethasone (dex) treatment in an ag mutant background $^{48}$ triggered a 2.5 -fold increase in YUC4 expression within $4 \mathrm{~h}$ (Fig. 2j). Likewise, CRC-GR induction by dex treatment in a crc mutant background ${ }^{17}$ triggered YUC4 expression (Fig. 2k). Furthermore, simultaneous activation of $A G$ and $C R C$ by dex in AG-GR CRC-GR double transgenic plants led to a significant increase in YUC4 expression compared to either of the parental lines (Fig. 2l). Taken together, these data suggest that AG and CRC synergistically regulated YUC4 expression.

CRC binds to YUC4 promoter and promotes auxin accumulation. Our combined results suggest that CRC may directly upregulate YUC4. To identify candidate YABBY-binding sites in the YUC4 promoter, we first performed phylogenetic shadowing
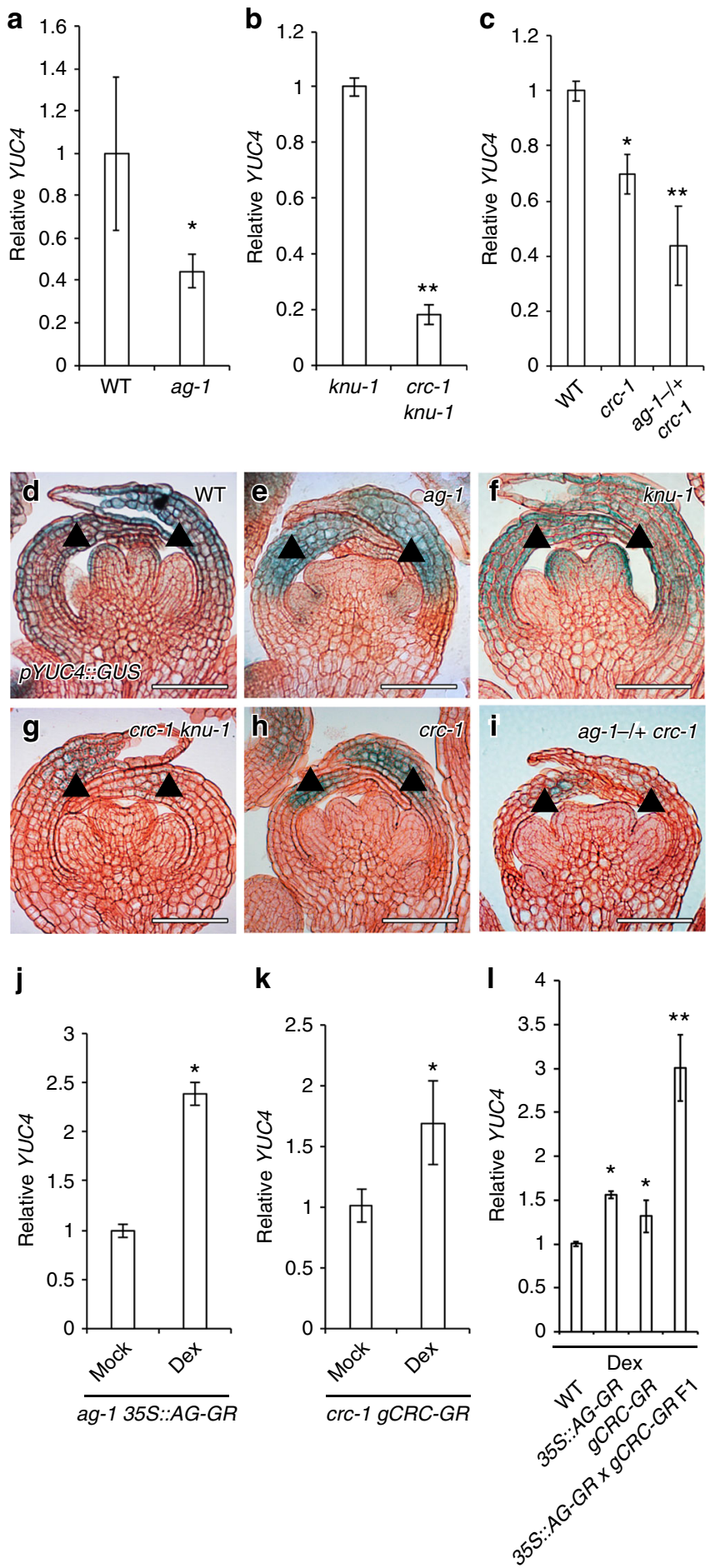

Fig. 2 AG and CRC synergistically control YUC4 expression. a-c YUC4 mRNA levels in wild-type and ag-1 (a), knu-1 and crc-1 knu-1 (b), wild-type, $\mathrm{crc}^{-1}$, and $a g-1-/+\mathrm{crc}^{-1}$ (c) floral buds. The values are represented as the means \pm SEMs. $p$-values were calculated with a two-tailed Student's $t$-test. ${ }^{\star} p<0.05$ compared to wild type. ${ }^{\star \star} p<0.05$ compared to single mutant. d-i pYUC4::GUS in wild-type (d), ag-1 (e), knu-1 (f), crc-1 knu-1 (g), crc-1 (h), and $\mathrm{ag}-1-/+\mathrm{Crc}-1$ (i) floral buds at the stage 6 . Arrowheads indicate abaxial side of epidermal cells in carpels at stage 6. $\mathbf{j}$-I YUC4 mRNA levels in mockand dex-treated ag-1 35 S::AG-GR (j), mock- and dex-treated crc-1 gCRC-GR (k), dex-treated wild-type, 35S::AG-GR, gCRC-GR, and 35S::AG-GR gCRC-GR F1 (I). The values are represented as the means \pm SEMs. $p$-values were calculated with a two-tailed Student's $t$-test. ${ }^{\star} p<0.05$ compared to mock or wild type. ${ }^{\star \star} p<0.05$ compared to single transgenic line. Bar $=50 \mu \mathrm{m}$ in $\mathbf{d}-\mathbf{i}$ 
a

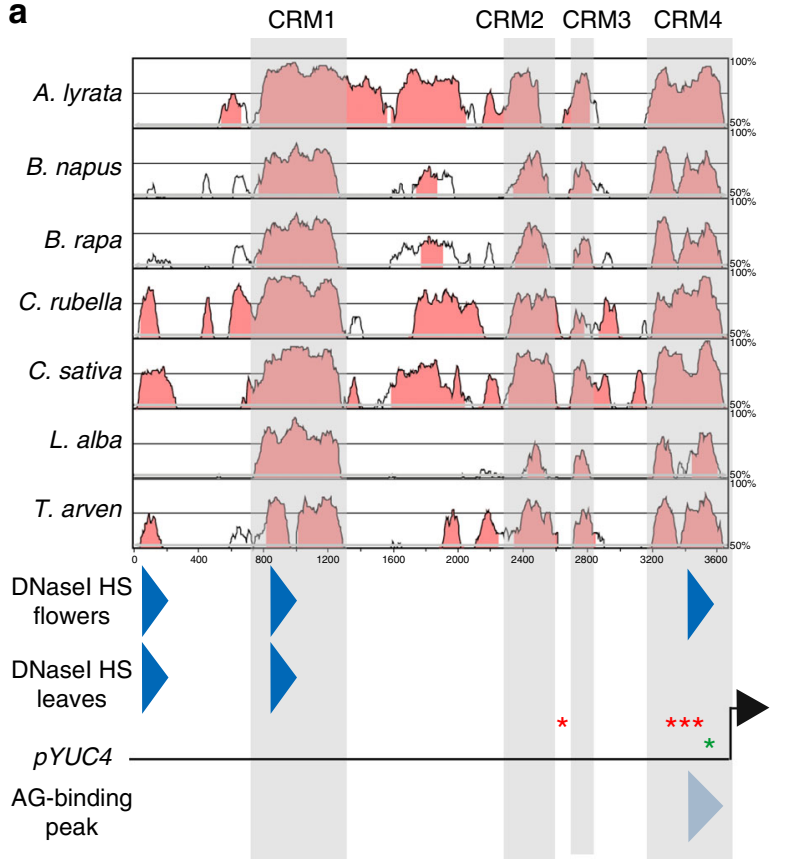

b
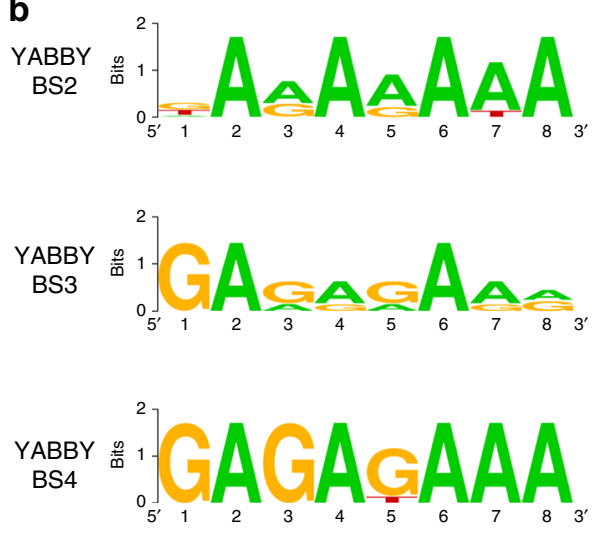
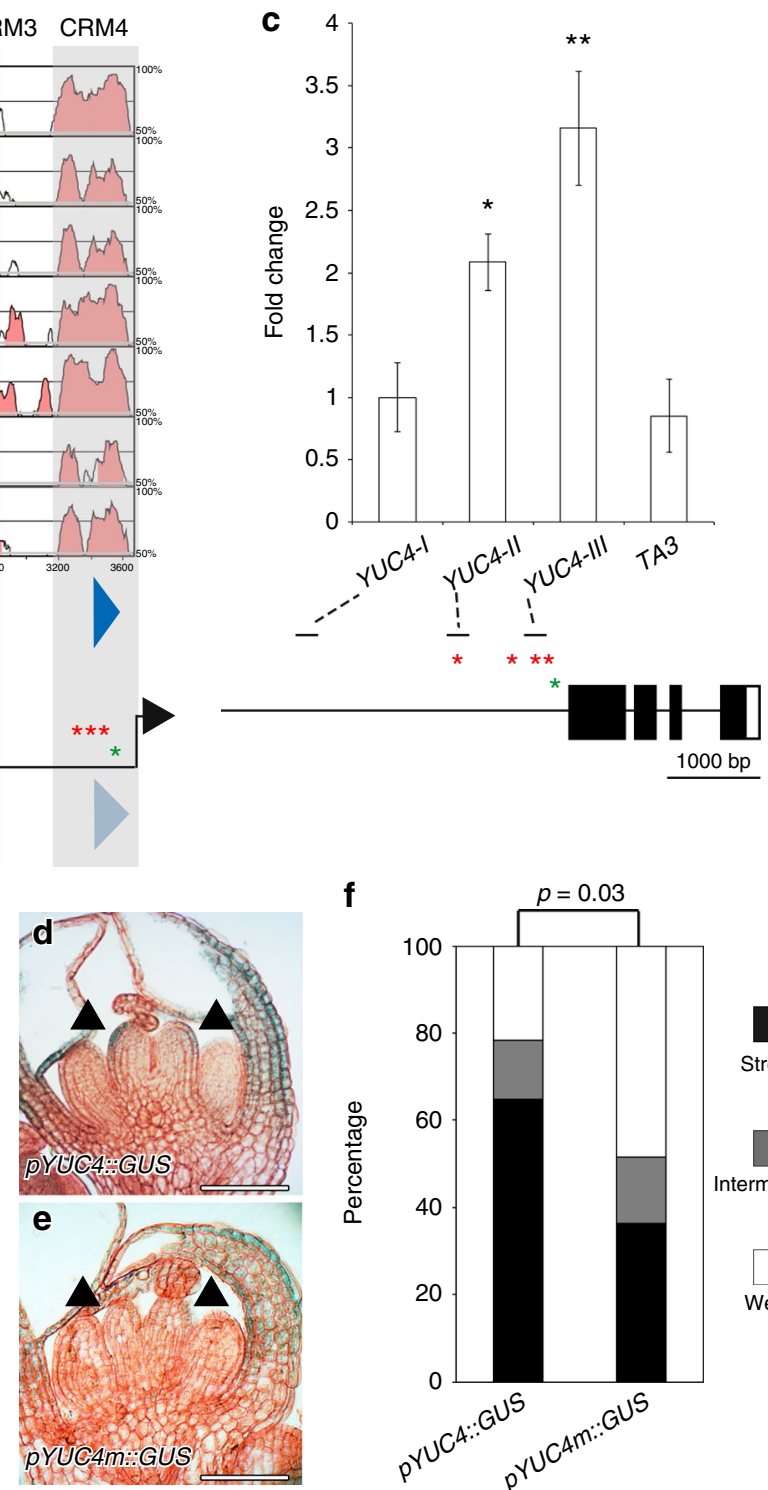

f
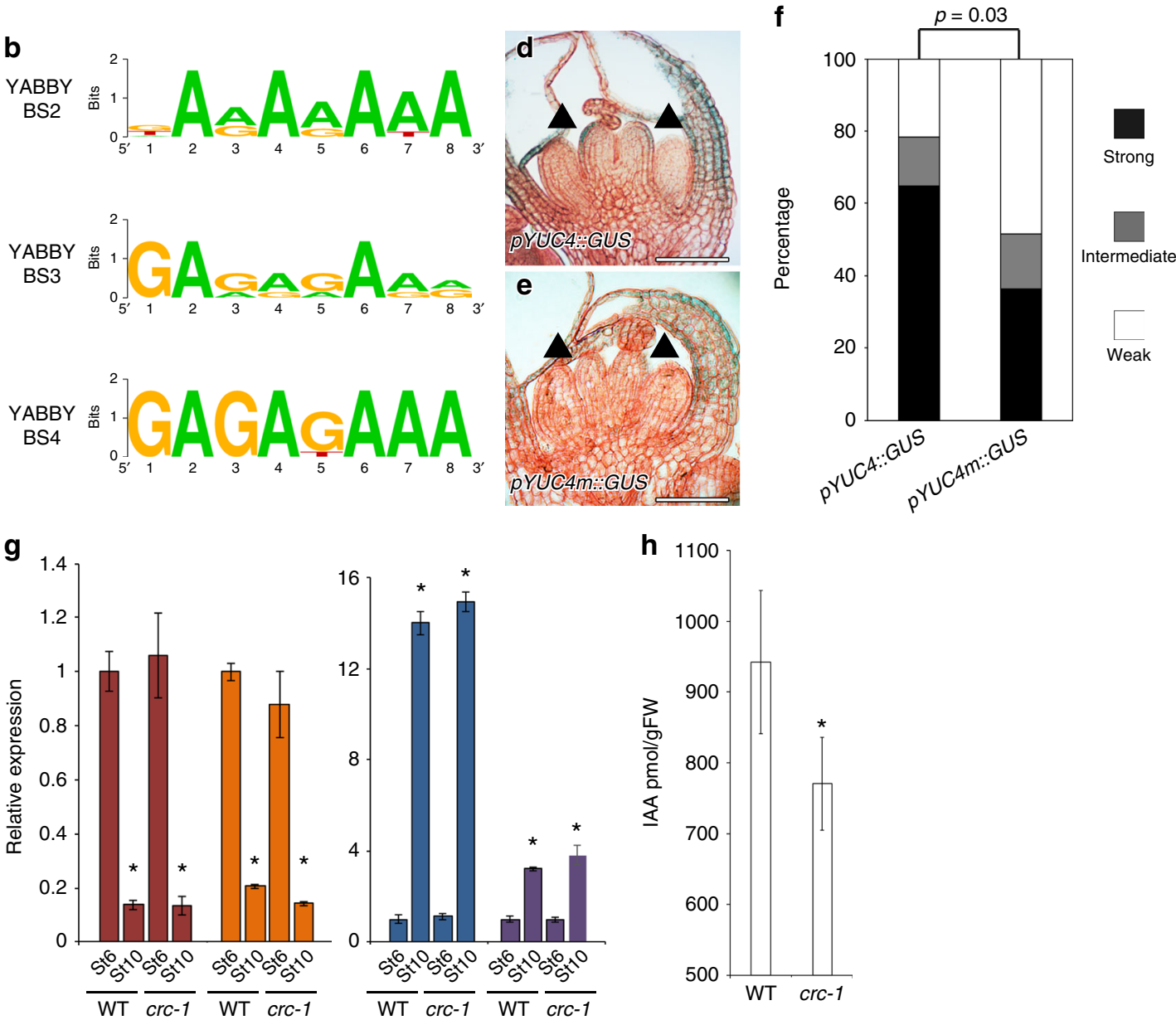

with seven Brassicaceae species ${ }^{15,17}$. Four conserved regulatory modules (CRMs) were identified and defined as CRM1, CRM2, CRM3, and CRM4 (Fig. 3a and Supplementary Fig. 7-9). Then, we identified DNase I hypersensitive sites where transcription factors could bind using published genome-wide data ${ }^{49}$ (Fig. 3a and Supplementary Fig. 10). CRM1 contained a DNase I hypersensitive site in the leaves and flowers, suggesting that CRM1 had a key role in regulating YUC4 expression in both vegetative and reproductive stages (Fig. 3a). CRM4 also contained a DNase I hypersensitive site that was observed only in the reproductive stage (Fig. 3a). Furthermore, an AG binding peak was in the CRM4. Within the peak, there is only one potential CArG box, where AG could bind (Fig. 3a). Since CRC is specifically expressed during the reproductive stage ${ }^{17}$, CRM4 might have an 
Fig. 3 CRC binds to the YUC4 promoter and controls auxin accumulation a Pairwise alignment created by mVISTA of the $5^{\prime}$ upstream intergenic region of the YUC4 promoter using seven Brassicaceae species. Four regions (gray: CRM1, CRM2, CRM3, and CRM4) are conserved in the YUC4 promoter. Blue triangles indicate DNase I hypersensitive sites. Red and green asterisks indicate CRC binding sites and a possible CArG box (C[C/T][A/T]G[A/G][A/ $\mathrm{T}]_{6}[\mathrm{~A} / \mathrm{G}] \mathrm{G}$ ) within an $A G$ binding peak, respectively. The binding region was shown by pale blue triangle. $\mathbf{b}$ Conservation of $C R C$ binding sites among Brassicaceae species. The diagram was generated by Weblogo. c Mapping of the CRC-myc protein to the YUC4 locus. ChIP using anti-myc in synchronized gCRC-myc floral buds at stage 6 in the ap1 cal background. The bottom section indicates the location of ChIP-qPCR amplicons tested. Red and green asterisks indicate CRC binding sites and possible CArG box, respectively. The values are represented as the means \pm SEMs. $p$-values were calculated with a two-tailed Student's $t$-test. ${ }^{\star} p<0.05$ compared to the TA3 signal. ${ }^{\star \star} p<0.01$ compared to the TA3 signal. $\mathbf{d}$, e Reporter gene expression in the wild-type and YABBY-binding site-mutated YUC4 promoter. GUS expression in stage 6 floral buds under control of the YUC4 promoter with (d) or without (e) intact YABBY-binding sites. Arrowheads indicate abaxial side of epidermal cells in carpels at stage 6 . $\mathbf{f}$ Visual scoring of $p$ YUC4::GUS or $p Y U C 4 m:: G U S$ staining in the T1 population; $n>32$ for each construct tested. $p$-values were calculated with a $\chi^{2} t$-test. $\mathbf{g}$ mRNA abundance of marker genes, $C L V 3$ (red), $L F Y$ (orange), PAL4 (blue), and COMT (purple) in wild-type and crc- 1 floral buds up to stage 6 and stage 10. The values are represented as the means \pm SEMs. $p$-values were calculated with a two-tailed Student's $t$-test. ${ }^{*} p<0.05$ compared to the signals at stage 6 in the same genetic backgrounds. $\mathbf{h}$ The amount of IAA in WT and crc-1 mutants determined by liquid chromatography-tandem mass spectrometry. Floral buds up to stage 8 were used for this assay. The values are represented as the means \pm SEMs. $p$-values were calculated with a two-tailed Student's $t$-test. ${ }^{*} p<0.05$ compared to the wild type. Bars $=50 \mu \mathrm{m}$ in $\mathbf{d}, \mathbf{e}$

important role in activating YUC4 expression. Consistent with this hypothesis, the deletion of CRM4 by genome editing led to partial reduction in YUC4 mRNA levels compared with the parental line (Supplementary Fig. 9). Remaining activity of YUC4 mRNA in the deletion line could be due to the presence of regulatory region other than CRM4. CRM4 contained three evolutionarily conserved YABBY-binding sites (GA[A/G]AGAAA) (Fig. 3a, b, and Supplementary Fig. 9). Two of these sites were highly conserved among all seven Brassicaceae species while the other was partially conserved only in four species (BS2). Although a fourth YABBY-binding site was observed in CRM2 and CRM3, it was not conserved. No other potential YABBY-binding sites $(\mathrm{CC}[\mathrm{C} / \mathrm{A}][\mathrm{T} / \mathrm{C}] \mathrm{C}[\mathrm{T} / \mathrm{A}][\mathrm{C} / \mathrm{T}] \mathrm{C}$ or $\mathrm{CCCCAC})$ were found anywhere in the YUC4 promoters among the seven Brassicaceae species $^{50}$ (Supplementary Fig. 7-10). Furthermore, no flowerspecific DNase I hypersensitive site containing YABBY-binding sites was found in the regulatory region of the three closest YUC4 homologs ${ }^{25}$ (YUC1, YUC2, and YUC6) (Supplementary Fig. 10).

To determine whether CRC directly binds to the YUC4 promoter, we used a genomic construct that expressed a CRCmyc fusion protein (gCRC-myc). Because of limited floral tissues at a specific stage, a ubiquitously expressed APETALA1 (AP1) fusion to GR in the ap1 cal mutant background was employed. The ap1 cal double mutant formed many floral primordia at the first and second developmental stages. The dex-induced nuclear entry of AP1-GR in the ap1 cal mutant resulted in the production of hundreds of floral stage-synchronized tissues ${ }^{51}$. Chromatin immunoprecipitation (ChIP) using synchronized CRC-myc flowers at stage 6 followed by qPCR revealed a strongest association of CRC-myc with the YUC4-III DNA fragment, which contained three evolutionarily conserved YABBY-binding sites found in CRM4 (Fig. 3a, c).

To test the biological functions of the YABBY-binding sites in the CRM4, all three YABBY-binding sites were disrupted in a full-length YUC4 promoter ( $p Y U C 4$ ), yielding $p Y U C 4 m$. In transgenic plants expressing GUS under control of $p Y U C 4 m$, GUS expression was specifically reduced in abaxial carpels at stage 6 of flower development (Fig. 3d, e). We analyzed the expression of these reporter constructs in an independent population of T1 plants $(n \geq 32)$ to minimize potential positional effects on transgene expression. Significantly more pYUC4m::GUS lines had weaker signals than $p Y U C 4:: G U S$ lines $(p=0.03)$ (Fig. 3f). The combined data suggest that CRC directly binds to the YUC4 promoter via highly conserved cis-elements.

Then, we quantified bioactive auxin to examine the contribution of the CRC-induced increase in YUC4 transcription to auxin accumulation. Since $C R C$ expression began in stage 6 and no morphological differences were observed between the wild type and $\operatorname{crc}$ single mutant at this stage ${ }^{13}$, flowers older than the stage 7 were manually selected for and removed using a dissecting microscope (Supplementary Fig. 11a-d). To evaluate the quality of these tissues, qRT-PCR was conducted. The expression of a stem cell marker gene (CLAVATA3; CLV3) and a young flowerspecific gene ${ }^{52,53}$ ( $L E A F Y$; $L F Y$ ) were higher in stage 6 floral buds than in stage 10 buds (Fig. 3g, and Supplementary Fig. 11e). By contrast, the transcript levels of genes involved in monolignol and cellulose biosynthesis, such as PHENTLALANINE AMMONIALYASE4 (PAL4) and CAFFEATE O-METHYLTRANSFERASE1 (COMT1), were very low in stage 6 tissues compared to stage 10 tissues $^{54}$ (Fig. 3g and Supplementary Fig. 11e). We used welltrimmed tissues to measure the amount of indole-3-acetic acid (IAA). Consistent with the observed reduction in YUC4 transcripts in the $c r c-1$ mutant, our analysis of IAA levels by mass spectrometry after liquid chromatography revealed that IAA levels were reduced in the $\operatorname{crc}$ mutants (Fig. 3h).

The AG complex controls chromatin accessibility at YUC4. One possible explanation for the synergistic activation of YUC4 by $A G$ and CRC could be a physical protein-protein interaction between AG and CRC. However, no interaction was observed between the two transcription factors based on yeast-two hybrid (Supplementary Fig. 12). Alternatively, chromatin accessibility at the YUC4 promoter may be affected. Since the imitation switch (ISWI)-type chromatin remodeling factors CHROMATIN REMODELING 11 (CHR11) and CHR17 were identified as AG interacting partners and a chr11 chr17 double mutant showed a split style phenotype ${ }^{55-58}$ (Supplementary Fig. 13), we examined the role of $A G$ and CHR11/CHR17 in regulating YUC4. We treated chr11 chr17 double mutants with exogenous auxin; however, no rescue was observed under our experimental conditions (Supplementary Fig. 13). Local accumulation of auxin is important for phenotypic rescue in $\operatorname{chr} 11 \mathrm{chr} 17$ mutants as is often seen in many mutants ${ }^{25}$. CHR11 and CHR17 were both expressed in terminating floral meristems at stage 6 (Fig. 4a, b). Based on qRT-PCR analysis, YUC4 expression was reduced in the chr11-1 chr17-1 double mutant compared to the wild type (Fig. 4c). We confirmed the reduction in YUC4 expression in the carpels of the chr11-1 chr17-1 double mutant at stage 6 (Fig. 4d, e). CHR11 bound to the proximal region of the YUC4 promoter, similar to AG (Fig. 4f and Supplementary Fig. 14).

The primary function of CHR11 and CHR17 is to slide nucleosomes and open up chromatin to allow proper gene expression $^{57,59}$. Once genes are transcriptionally activated, nucleosome occupancy typically decreases ${ }^{60}$. Published genomewide nucleosome distribution in the wild type revealed that there was one nucleosome upstream ( -1 nucleosome) and several well- 

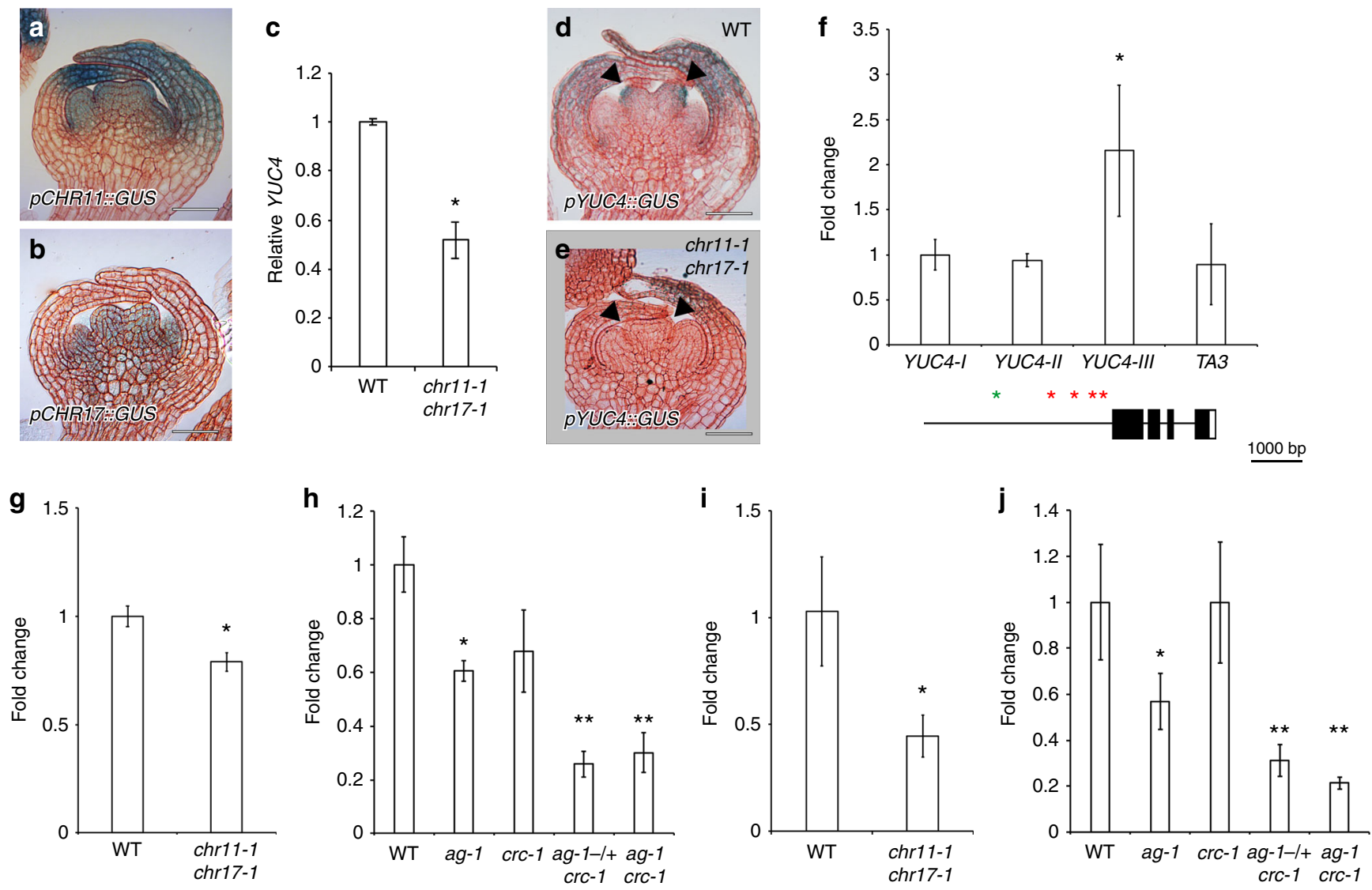

Fig. 4 AG and CRC are required for increased chromatin accessibility at the YUC4 loci. a, b Expression of $p$ CHR11::GUS (a) and pCHR17::GUS (b) in stage 6 floral buds. c YUC4 mRNA levels in wild-type and chr11-1 chr17-1 floral buds. The values are represented as the means \pm SEMs. $p$-values were calculated with a two-tailed Student's $t$-test. ${ }^{*} p<0.05$ compared to the wild type. d, e Expression of $p$ YUC4::GUS in wild-type (d) and chr11-1 chr17-1 (e) floral buds at the stage 6. Arrowheads indicate abaxial side of epidermal cells in carpels at stage 6. $\mathbf{f}$ Top: Mapping of the GFP-CHR11 protein association to the YUC4 locus. A GFP antibody was used to detect CHR occupancy. Bottom: Diagram of ChIP-qPCR amplicons tested. Red and green asterisks indicate CRC binding sites and possible $C A r G$ box $(C[C / T][A / T] G[A / G][A / T] 6[A / G] G)$ within an $A G$ binding peak, respectively. The values are represented as the means \pm SEMs. $p$-values were calculated with a Student's t-test. *: $p<0.05$ compared to the TA3 signal. $\mathbf{g}$, $\mathbf{h}$ Chromatin accessibility at the YUC4 locus. $\mathbf{g}$ ) Wild-type and chr11-1 chr17-1 floral buds. h) Wild-type, ag-1, crc-1, ag-1-/ + crc-1, and ag-1 crc-1 floral buds. The values are represented as the means \pm SEMs. $p$-values were calculated with a two-tailed Student's $t$-test. ${ }^{\star} p<0.05$ compared to the wild type. ${ }^{\star \star} p<0.05$ compared to single mutants. i, j RNA Pol II association with the YUC4 locus. A Pol II antibody was used. i Wild-type and chr11-1 chr17-1 floral buds. j Wild-type, ag-1, crc-1, ag-1-/+ crc-1, and ag-1 crc-1 floral buds. The values are represented as the means \pm SEMs. $p$-values were calculated with a two-tailed Student's $t$-test. ${ }^{\star} p<0.05$ compared to the wild type. ${ }^{\star *} p<0.05$ compared to single mutants. Bars $=50 \mu \mathrm{m}$ in $\mathbf{a}, \mathbf{b}, \mathbf{d}, \mathbf{e}$

positioned nucleosomes downstream of the YUC4 transcriptional start site (TSS) (Supplementary Fig. 15) ${ }^{57}$. Consistent with the observed reduction in YUC4 expression in the chr11 chr17 double mutant, published MNase-seq data ${ }^{57}$ show a higher nucleosome occupancy $(+1$ nucleosome) on the TSS of YUC4 compared to the wild type (Supplementary Fig. 15). To further examine the effect of CHR11/CHR17, AG, and CRC on the accessibility of the YUC4 locus, we performed Formaldehyde Assisted Isolation of Regulatory Elements (FAIRE) ${ }^{61}$. The FAIRE assay revealed a reduction in open chromatin at the YUC4 promoter in the chr11 chr17 double mutant compared to the wild type (Fig. 4g). Likewise, the ag mutation decreased accessibility to YUC4 based on FAIRE (Fig. 4h). On the other hand, we did not see statistic difference in chromatin accessibility between wild type and $\mathrm{crc}$ mutant (Fig. 4h). A further reduction in chromatin accessibility was observed in the ag-1-/+ $c r c-1$ heterozygote and ag-1 $c r c-1$ double mutant compared to either single mutant (Fig. 4h). RNA polymerase II (Pol II) occupancy was also reduced in the chr11 chr17 mutant relative to the wild type (Fig. 4i). A similar reduction in Pol II occupancy was observed in the ag-1 mutant relative to the wild type (Fig. 4j). By contrast, no difference in Pol
II occupancy was observed in the $\operatorname{crc}-1$ mutant (Fig. 4j), and a subtle reduction was observed in the ag-1 mutant compared with the wild type. In addition, a further reduction was observed in the ag-1-/+ crc-1 heterozygote and the ag-1 crc-1 double mutant compared to the parental lines (Fig. 4j). Taken together with expression analyses, our results may suggest that the AG-CHR11/ CHR17 complex controls chromatin-mediated accessibility to the YUC4 promoter and thereafter CRC affects the chromatin structure through transcriptional regulation.

YUC4 misexpression rescues the indeterminacy in crc $k n u$. Since YUC4 expression is directly controlled by CRC, we then investigated whether expressing YUC4 was sufficient to rescue the crc mutant phenotype (Fig. 5a-n). We expressed YUC4 under the control of the CRC promoter in the crc mutant and sensitized crc knu mutant. As previously reported ${ }^{13,17}$. crc mutants had shorter and wider fruits than the wild type (Fig. 5a, b, g, h). Furthermore, $\operatorname{crc}$ mutants failed to fuse at the fruit tips and had shorter styles than the wild type (Fig. $5 \mathrm{a}, \mathrm{b}, \mathrm{g}, \mathrm{h}, \mathrm{m}$ ). The $\mathrm{crc}$ mutant phenotype was significantly rescued by introducing YUC4 under the control of the $C R C$ promoter $\left(p=9.5 \times 10^{-13}\right.$; Fig. $5 b$, 

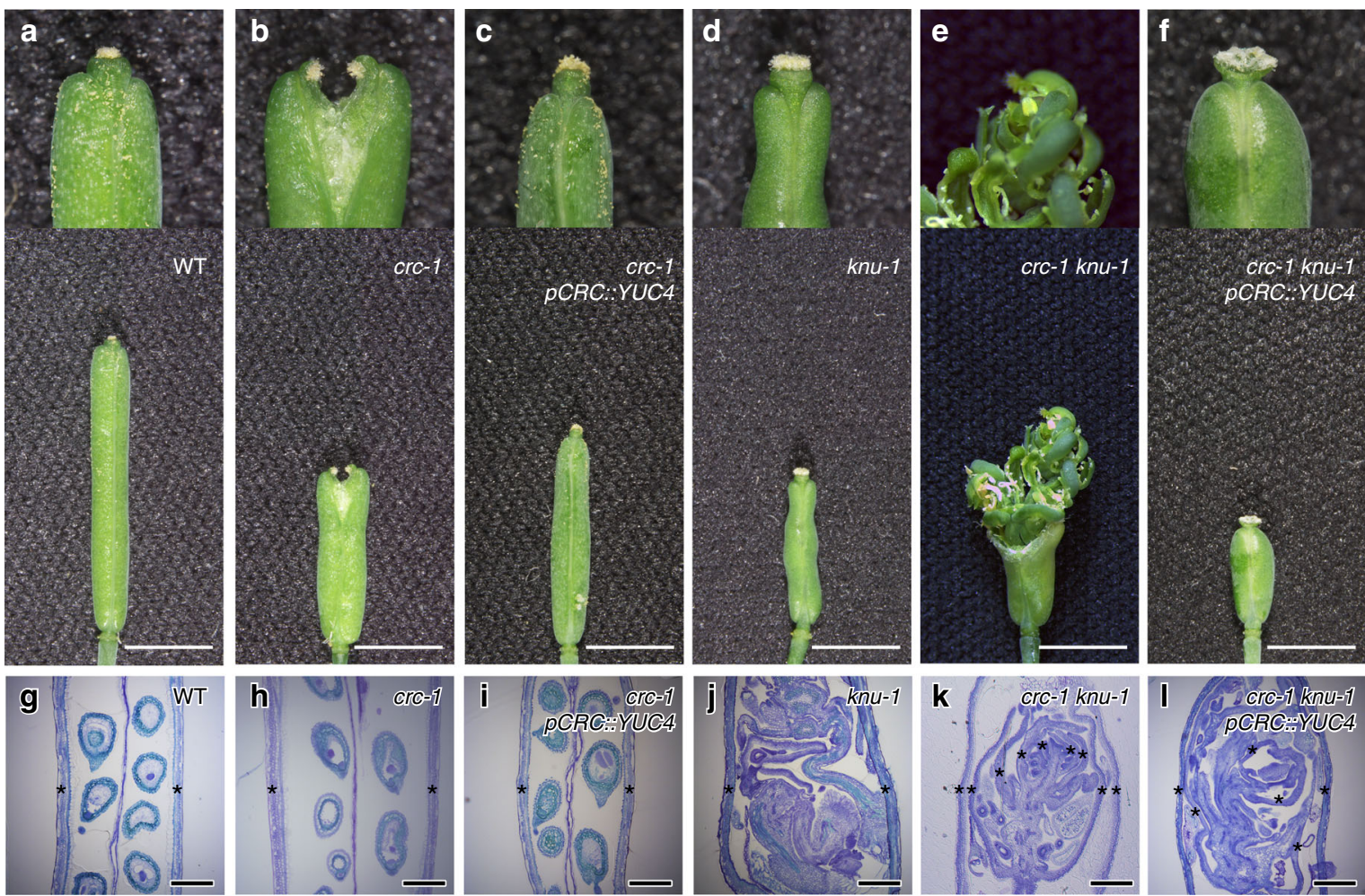

m

$p=9.5 \times 10^{-13}$

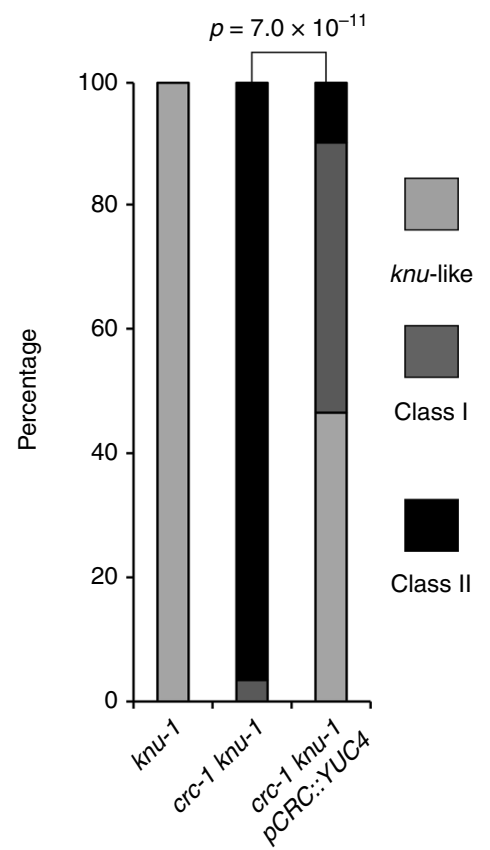

n
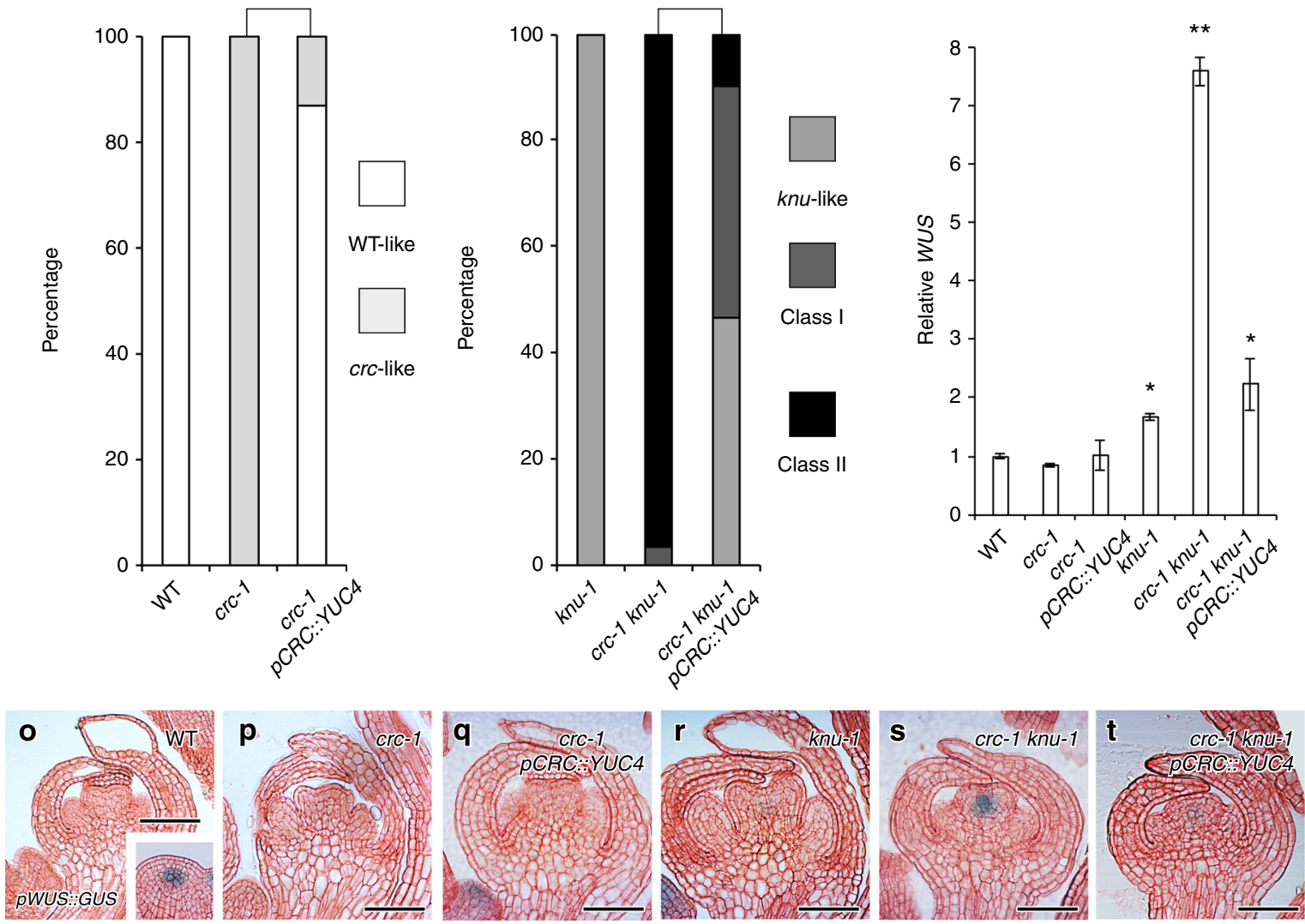

c, h, i, m). The knu-1 and crc-1 knu-1 mutants formed bulged gynoecia that contained additional whorls of carpels, and the indeterminate shoot phenotype, respectively ${ }^{17}$ (Fig. 5d, e, j, k). When $c r c k n u$ was crossed with $p C R C:: Y U C 4$, the resulting plants ( $p C R C:: Y U C 4$ crc knu) did not exhibited outgrowth of the indeterminate shoot phenotype (Fig. $5 d-f, j-1)$. Misexpression of YUC4 under control of the CRC promoter in the crc knu mutant significantly rescued the effect of the $\operatorname{crc}$ mutation $(p=7.0 \times 10$ $-11)$ : approximately half of the $p C R C:: Y U C 4$ crc- 1 knu-1 fruits showed a knu mutant-like phenotype. These results indicated 
Fig. 5 Rescue of indeterminacy in crc knu by ectopic expression of YUC4. a-f Morphology of wild-type (a), crc-1 (b), crc-1 pCRC::YUC4 (c), knu-1 (d), crc-1 knu-1 (e), and crc-1 knu-1 pCRC::YUC4 (f) fruits. Above: Close-up views of fruit tips. Below: Shapes of whole fruits. Arrowheads indicate stigma structures. g-I Longitudinal section of wild-type (g), crc-1 (h), crc-1 pCRC::YUC4 (i), knu-1 (j), crc-1 knu-1 (k), and crc-1 knu-1 pCRC::YUC4 (I) fruits. Asterisks indicate carpels. $\mathbf{m}$ Quantification of the mutant phenotype. $p$-values were calculated with a Chi-Square test. $\mathbf{n}$ Expression of the stem cell marker WUS in wild-type, crc-1, crc-1 pCRC::YUC4, knu-1, crc-1 knu-1, and crc-1 knu-1 pCRC::YUC4 flowers. The values are represented as the means \pm SEMs. $p$-values were calculated with a two-tailed Student's $t$-test. ${ }^{\star} p<0.05$ compared to the wild type. ${ }^{\star \star} p<0.05$ compared to the knu-1 mutants. o-t Expression of the stem cell marker WUS in longitudinal sections of wild-type (o), crc-1 (p), crc-1 pCRC::YUC4 (q), knu-1 (r), crc-1 knu-1 (s), and crc-1 knu-1 pCRC::YUC4 (t) stage 6 floral buds. Images are shown at the same magnification. Bars $=1 \mathrm{~cm}$ in $\mathbf{a}-\mathbf{f} ; 500 \mu \mathrm{m}$ in $\mathbf{g}-\mathbf{l} ; 50 \mu \mathrm{m}$ in o-t

that local overproduction of YUC4 in the CRC expression domain was sufficient to restore the $\operatorname{crc} k n u$ mutant back to the knu phenotype.

To investigate the effect of YUC4 misexpression on the floral meristem indeterminacy phenotype at molecular level, we examined the expression of a stem cell determinant gene, WUS (Fig. $5 n-t)$. Although a strong pWUS::GUS signal was detected in the inflorescence meristem in wild-type plants, we could not detect the signal in the wild type or $\operatorname{crc}-1$ floral buds at stage 6 (Fig. 5n-p). No WUS signal was observed in $c r c-1$ pCRC::YUC4, which was consistent with the phenotypic similarity with wildtype plants (Fig. 5n, o, q). In knu and crc knu stage 6 mutant flowers, WUS was either weakly or strongly misexpressed, respectively ${ }^{11,17}$ (Fig. 5n, r, s). The strong WUS expression in crc knu was compromised when we introduced pCRC::YUC4 (Fig. 5n, s, t). Our combined data implied that YUC4 misexpression restored the crc knu mutant phenotype and WUS expression to that of the $k n u$ mutant.

YUC4 misexpression rescues the cell wall defects in crc knu. A microarray analysis suggested that floral meristem termination could be associated with cell wall remodeling (Fig. 1). To examine the effect of the AG-CRC-YUC4 pathway on cell wall components during floral meristem termination, we probed stage 6 floral bud sections with antibodies that recognized specific plant cell wall components. In the wild-type inflorescence meristem and floral meristem, immunolocalization of methyl esterified homogalacturonan (HG) with JIM7 exhibited a uniform labeling pattern over the different floral organs throughout cell walls ${ }^{30}$ (Fig. 6a and Supplementary Fig. 16). No difference was found in methyl esterified HG distribution using JIM7 between the mutants or transgenic plants and the wild type (Fig. 6a-f).

LM5 specifically recognizes (1-4)- $\beta$-D-galactan. As previously reported on the inflorescence meristems, this antibody binds weakly to the L1 layer cells of terminating floral meristems in the wild type $^{30}$ (Fig. $6 \mathrm{~g}$ and Supplementary Fig. 16). A similar LM5 labeling pattern and signal intensity was observed in $\operatorname{crc}-1, \mathrm{crc}-1$ pCRC::YUC4, and knu-1 (Fig. 6h-j), and the LM5 labeling intensity was reduced in the $c r c-1$ knu-1 double mutants (Fig. 6k). Consistent with the phenotypic rescue of crc knu by YUC4 misexpression, labeling intensity with LM5 in $\mathrm{crc} k n u$ pCRC:: YUC4 was also restored (Fig. 61, Supplementary Fig. 16). Xyloglucan recognized by LM15 was weakly observed in the L1 layer of the wild-type inflorescence meristem, where it accumulated uniformly in the developing carpels ${ }^{30}$ (Fig. $6 \mathrm{~m}$ and Supplementary Fig. 16). A similar LM15 binding pattern was observed in $c r c-1, k n u-1$, and pCRC::YUC4 (Fig. 6n-p); however, xyloglucan accumulation in the L1 layer of the developing $c r c-1$ $k n u-1$ carpels was weaker than in the wild type (Fig. 6q). This reduction was also partially restored by ectopic expression of YUC4 (Fig. 6r). Since we did not observe differences in the thickness of the cell wall in any carpel primordia among the tested lines, alterations in our antibody labeling experiment were largely due to changes in cell wall composition (Supplementary Fig. 17).
YUC4 and TRN2 regulate floral meristem determinacy. To examine whether YUC4 and TRN2 both control floral meristem determinacy downstream of CRC, we ectopically expressed YUC4 and removed TRN2 activity in the crc knu double mutant background (Fig. 7a-1). Although trn 2 fruits were twisted, the mutant did not show defects in floral meristem determinacy (Fig. 7b, f). The effect of the $\mathrm{crc}$ mutation on floral meristem indeterminacy in the $k n u$ mutant background was partially restored by the introduction of the trn 2 mutation $\left(p=1.9 \times 10^{-3}\right)$ (Fig. $7 \mathrm{a}-\mathrm{c}, \mathrm{e}-\mathrm{g}$, i) as previously reported ${ }^{17}$. Activation of YUC4 in the crc knu mutant background also partially restored the mutant phenotype to that of the $k n u$ mutant $\left(p=1.1 \times 10^{-10}\right)$. Simultaneous activation of YUC4 and removal of TRN2 led to a dramatic rescue of the effect of the crc mutation to that of the $k n u$ mutant background ( $p=2.4 \times 10^{-13}$ ) (Fig. $\left.6 \mathrm{~d}, \mathrm{~h}, \mathrm{i}\right)$. Less than $5 \%$ of the crc knu trn2 pCRC::YUC4 plants showed the strong crc knu mutant phenotype. Most $c r c-1$ knu-1 trn2-1 pCRC::YUC4 fruits showed the $k n u-1$ phenotype in terms of floral meristem determinacy (Fig. 7d, h, i). The crc-1 knu-1 trn 2-1 pCRC::YUC4 fruits never showed unclosed fruits phenotype which often seen in $\mathrm{crc}-1$ knu-1 trn2-1. Inside structure in crc-1 knu-1 trn2-1 pCRC::YUC4 and $k n u-1$ was similar to each other (Figs. $5 \mathrm{j}$ and $7 \mathrm{~h}$ ).

To test whether the phenotypic changes seen in crc-1 knu-1 trn2-1 pCRC::YUC4 were associated with WUS expression, we examined WUS expression by qRT-PCR. The strong WUS expression in the crc knu double mutants was attenuated by either activation of YUC4 or removal of TRN2 ${ }^{17}$ (Fig. 7j). We observed a further reduction of WUS expression in $c r c-1 \mathrm{knu}-1$ trn2-1 pCRC::YUC4 compared to $\mathrm{crc}-1 \mathrm{knu}-1 \mathrm{trn} 2-1$ or $\mathrm{crc}-1 \mathrm{knu}-$ 1 pCRC::YUC4. Hence, the two direct CRC targets, YUC4 and TRN2, cooperatively contribute to the termination of floral meristem development through WUS regulation (Fig. 7k).

\section{Discussion}

The timing of floral meristem termination is critical to forming a female reproductive structure (gynoecium). Despite the importance of gynoecium development for reproductive success, little is known about the switch from floral stem cell maintenance to gynoecium formation that is induced by the plant hormone auxin. Here, we showed that the auxin biosynthesis gene, YUC4, contributes to the transition from floral stem cell maintenance to gynoecium formation in Arabidopsis. Two tissue-specific transcription factors, AG and CRC, bind directly to the YUC4 promoter and activate its expression. Positive transcriptional feedforward loops, where a transcription factor directly activates another transcription factor, have been observed during flower development and auxin synthesis ${ }^{62,63}$. Although the CArG box and YABBY-binding sites (CRC binding site) are located close to each other on the YUC4 promoter, we did not observe protein-protein interactions between $A G$ and $C R C$. Instead, we found that feed-forward activation of YUC4 expression by AG and CRC directs the coordination of a precise change in chromatin state. This feed-forward loop may be important to overcome polycomb-mediated silencing and to reactivate the YUC4 gene only in correct timing and place $^{64}$. Previous proteomics approaches identified some nucleosome-associated factors that physically interact with $\mathrm{AG}^{58}$. Among these nucleosome- 

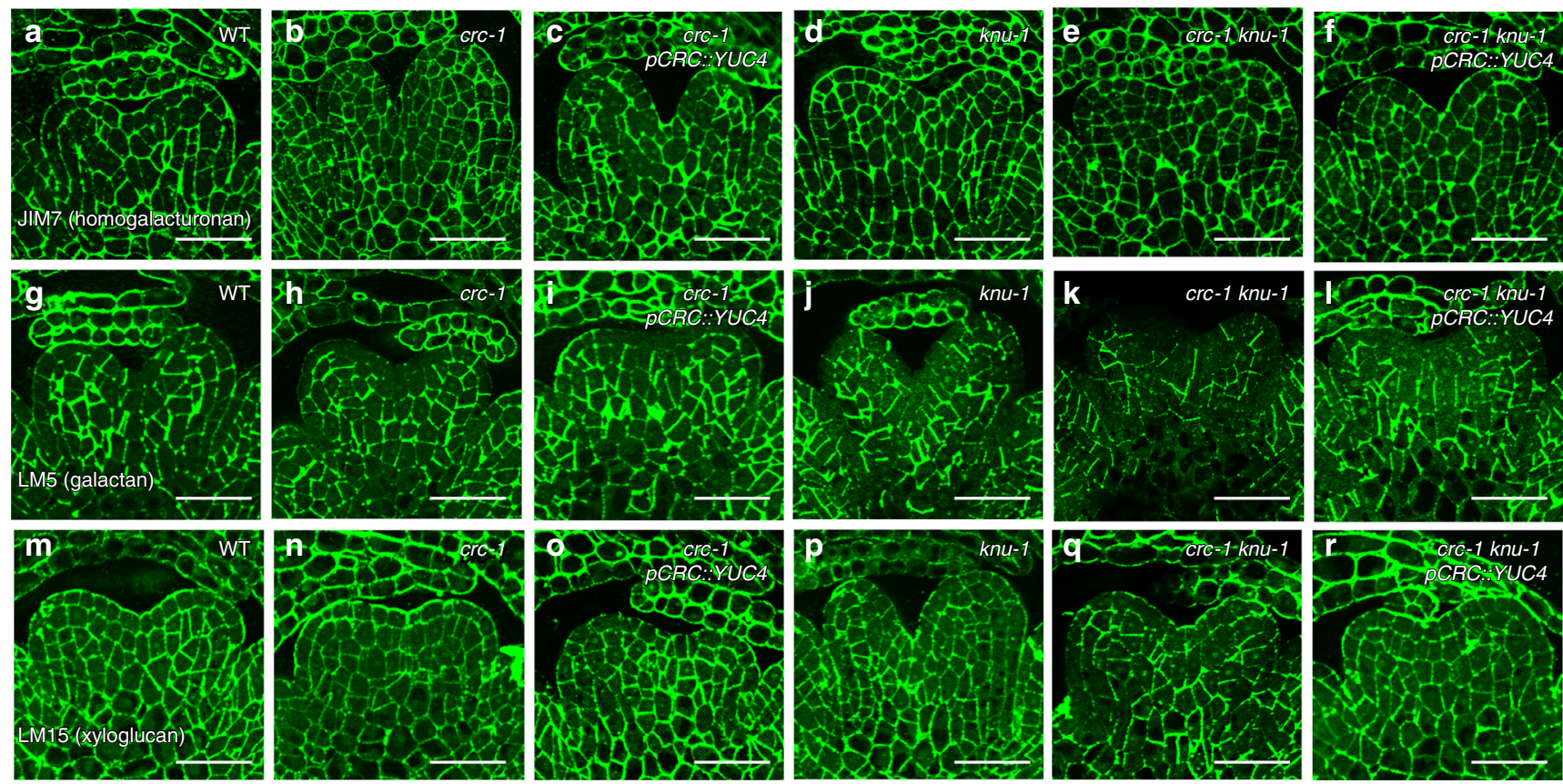

Fig. 6 Rescue of galactan and xyloglucan distribution in the crc knu by ectopic expression of YUC4. a-f Immunolocalization of homogalacturonan epitopes with JIM7 using longitudinal sections of wild-type (a), crc-1 (b), crc-1 pCRC::YUC4 (c), knu-1 (d), crc-1 knu-1 (e), and crc-1 knu-1 pCRC::YUC4 (f) stage 6 floral buds. g-I Immunolocalization of (1-4)-b-D-galactan epitopes with LM5 using longitudinal sections of wild-type (g), crc-1 (h), crc-1 pCRC::YUC4 (i), knu-1 (j), crc-1 knu-1 (k), and crc-1 knu-1 pCRC::YUC4 (I) stage 6 floral buds. m-r Immunolocalization of xyloglucan epitopes with LM15 using longitudinal sections of

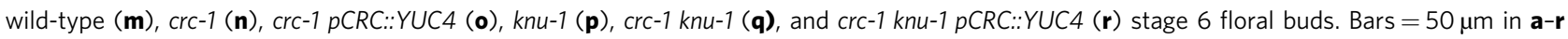

associated factors, SWI2/SNF2 chromatin remodeling ATPase is known to alter the interaction between the histone octamer and the DNA using ATP hydrolysis for energy ${ }^{65}$. The ISWI-type chromatin remodeling factors CHR11 and CHR17 were shown to fuse carpels ${ }^{58}$. Since $a g$ and $c h r 11 \mathrm{chr} 17$ mutants displayed similar levels of YUC4 expression, Pol II binding, and chromatin structure, CHR11 and CHR17 may enable AG to activate its targets. Since previous studies and our analyses agree that expression of $A G$ and $C H R 11 / C H R 17$ overlap during flower development stage $3^{3,56}$ (Supplementary Fig. 18), we propose that the AG-CHR chromatin remodeling complex opens up compacted chromatin, thereby increasing accessibility to the YUC4 gene prior to floral meristem termination. Although YUC4 expression was reduced in the ag mutant, no difference was reported in floral meristem size between the wild type and ag mutant by stage $3^{66}$. This could be due to redundant activity within the YUC gene family or other auxin-related regulators such as TRN2 (see below). Except for YUC4, none of the other YUC family members have been identified as direct AG targets according to previous genome-wide AG binding data ${ }^{40}$. We also did not detect any differences in the expression of other YUC genes between the wild type and ag-1 mutants (Supplementary Fig. 19).

Because CRC expression starts in stage 6 of flower development, it is most likely that CRC binds to the YUC4 promoter after the AG-CHR11/CHR17 complex opens up the YUC4 locus $3,13,57,58$. Thus, a synergistic activation of YUC4 is more likely to occur where and when both $A G$ and $C R C$ are expressed. High expression of YUC4 was only detected in the abaxial region of carpel primordia at stage 6. At the molecular level, the AGCHR chromatin remodeling complex may allow additional transcription factors, such as CRC, to access their respective ciselements. This could lead to further recruitment of general transcriptional machinery, including RNA Pol II and possibly other Pol II complex components, to boost transcription of YUC4 (Fig. 7l). Although we did not observe a difference in $A G$ expression between the wild type and chr11-1 chr17-1 double mutant, CRC expression was decreased in the chr11-1 chr17-1 double mutant (Supplementary Fig. 13). Thus, the AG-CHR11/ CHR17 complex might control CRC expression in addition to YUC4 expression. The synergistic feed-forward regulation of YUC4 expression by AG and CRC directs the precise chromatin state switch from floral stem cell maintenance to gynoecium formation through auxin. Furthermore, auxin maxima are likely established in the terminating floral meristem during stage 6, together with other auxin synthesis enzymes (YUCs, TAA) and the CRC-mediated auxin homeostasis regulator TRN217,24 Indeed, the indeterminate phenotype of the yuc4 mutant was only observed in the sensitized $y u c$ multiple mutant background ${ }^{25}$.

Previously, we reported that CRC terminates floral meristem through the auxin homeostasis pathway ${ }^{17}$. The CRC downstream target, TRN2, contributes to this termination, at least partially ${ }^{17}$. Here, we showed that two auxin-related CRC targets, YUC4 and $T R N 2$, function in a parallel pathway for the termination of floral meristem. In the crc knu double mutant, YUC4 expression decreased, whereas TRN2 expression increased. Ectopic expression of YUC4 and removal of TRN2 in the crc knu background resulted in a phenotype similar to that of the $k n u$ mutant. Since we observed significant rescue of floral meristem determinacy (that had been disrupted by the $\operatorname{crc}$ mutation) in the sensitized knu mutant background, these two genes could be the major auxin homeostasis targets that act downstream of CRC.

We also noticed a difference in the accumulation of galactan and xyloglucan in the cell wall of the crc knu double mutant compared to the wild type. Galactan accumulated at lower levels in the L1 layer in the wild-type shoot apical meristem and terminating floral meristem at the stage 6 . This suggested that the high mobility of galactan in the cell wall and/or reversible binding of galactan to cellulose in a layer-specific manner might be important for proper floral meristem function, as was previously suggested for the shoot apical meristem 67 . In addition to the defects in floral meristem activity, galactan levels were also reduced in all three floral meristem layers of the crc knu double 

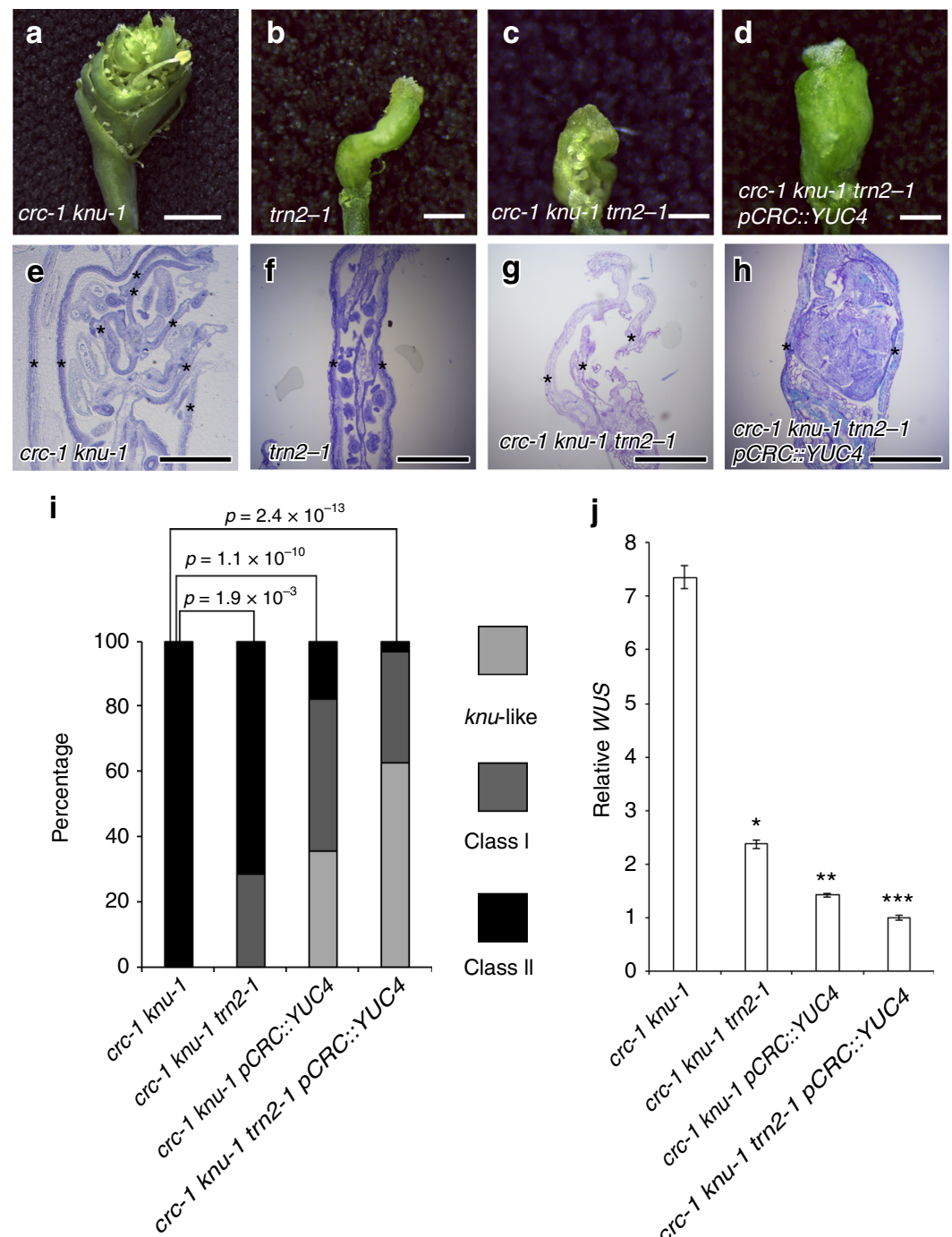

j

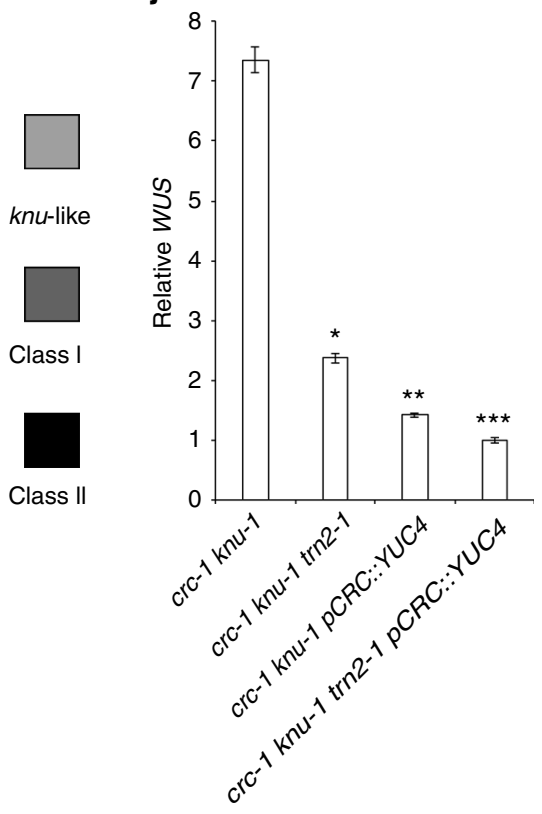

$\mathbf{k}$
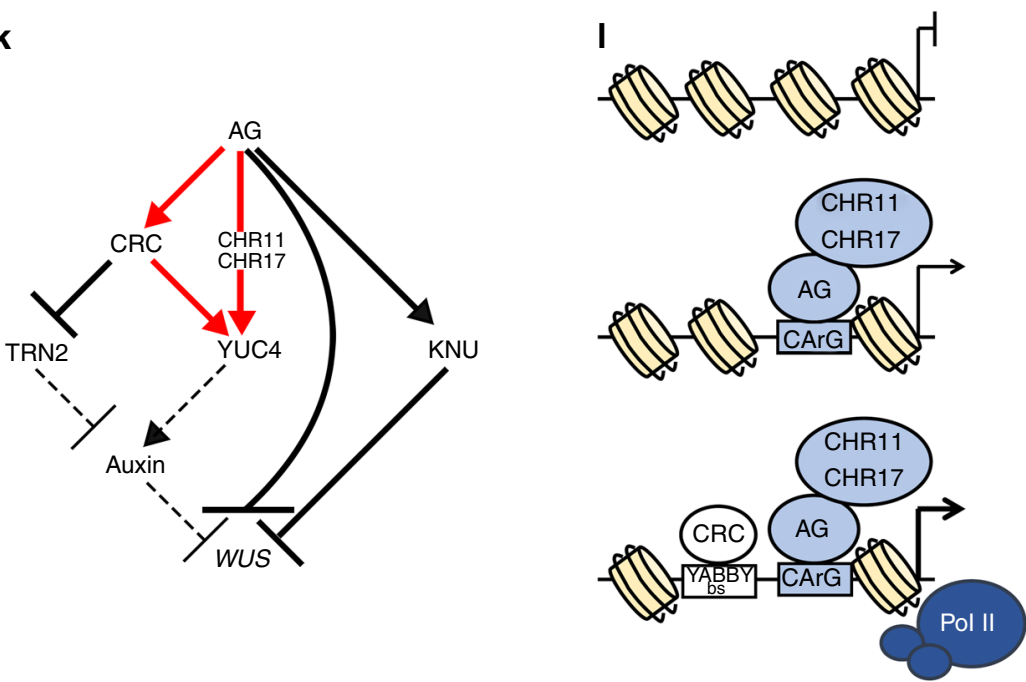

mutant. Xyloglucan also accumulated in a layer-specific manner in the wild-type shoot apical meristem, but not in the wild-type floral meristem at stage $6^{30}$. Interestingly, tissue-specific accumulation of xyloglucan was only observed in the crc knu floral meristem at the stage 6 . This finding suggests that cellular morphogenetic events might be similar between the wild-type shoot apical meristem and the crc knu mutant floral meristem. Our antibody labeling of wall components suggests a potential role for galactan and xyloglucan rearrangements in floral meristem termination. Furthermore, alterations in cell wall composition in the $\mathrm{crc} k n u$ mutant were partially rescued when YUC4 was misexpressed. These data suggest that the cell wall composition 
Fig. 7 Rescue of the crc knu mutant phenotype by modulating two CRC downstream target genes. a-d Morphology of crc-1 knu-1 (a), trn2-1 (b), trn2-1 crc-1 knu-1 (c), and trn2-1 crc-1 knu-1 pCRC::YUC4 (d) fruits. e-h Longitudinal section of crc-1 knu-1 (e), trn2-1 (f), trn2-1 crc-1 knu-1 (g), and trn2-1 crc-1 knu-1 pCRC:: YUC4 (h) fruits. Asterisks indicate carpels. i Quantification of mutant phenotypes of crc-1 knu-1, crc-1 knu-1 trn2-1, crc-1 knu-1 pCRC::YUC4 and crc-1 knu-1 trn2-1 pCRC::YUC4 fruits. $p$-values were calculated with a Chi-Square test. $\mathbf{j}$ Expression of the stem cell marker WUS in crc-1 knu-1, crc-1 knu-1 trn2-1, crc-1 knu-1 pCRC::YUC4, and crc-1 knu-1 trn2-1 pCRC::YUC4 flowers. $p$-values were calculated with a two-tailed Student's $t$-test. ${ }^{\star} p<0.05$ compared to crc knu. ${ }^{\star \star} p$ $<0.05$ compared to crc knu trn2. ${ }^{* \star} p<0.05$ compared to crc knu $p C R C:: Y U C 4$. k Regulatory network for AG-mediated floral meristem termination in Arabidopsis. Red arrows: feed-forward regulation of the YUC4 by AG and CRC. Solid arrows: direct transcriptional regulation. Dashed arrows: relationship may not be direct. I A model for the YUC4 transcription. Bars $=3 \mathrm{~mm}$ in $\mathbf{a} ; 1 \mathrm{~mm}$ in b-d; $500 \mu \mathrm{m}$ in $\mathbf{e}-\mathbf{h}$

downstream of CRC might be regulated by auxin. A second possibility is that this rescue reflects the ability of auxin biosynthesis to partially bypass defects in cell wall composition. In this scenario, CRC target candidates, such as EXP8 and Atc$w I N V 1$, could have important roles in cell wall composition.

\section{Methods}

Plant materials and growth condition. All plants used in this study were in the Landsberg erecta (Ler) background, except for chr11-1 chr17-1 and pTAA1::TAA1GFP, which were in the Columbia (Col) background. The ag-1, crc-1, knu-1, crc-1 knu-1, crc-1 gCRC-GR, ag-1 35S::AG-GR, ap1 cal-1 35S::AP1-GR gCRC-myc, chr111 chr17-1, pTAA1::TAA1-GFP, pWUS::GUS, and trn2-1 plants were previously described $^{9,13,17,20,24,48,51,56}$. For plant materials at the mature flower stage, seeds were sown on vermiculite and Metro-Mix. For antibiotic selection, seeds were sown on half-strength Murashige and Skoog plates. All plants were grown at $22{ }^{\circ} \mathrm{C}$ under 24-h light conditions.

Comparison of transcriptome datasets. Four available transcriptome datasets were used to identify genes that are regulated by the AG and CRC $6,17,34$. Genotypes and data sources are in Supplementary Table 1. Published lists of differentially expressed genes were used for $a g-11$ ap2-35 and $a g-11$ ap2-43 mutants ${ }^{6}$. For the $a g$ 12 mutant, raw data were downloaded from TAIR and analyzed using BIOCONDUCTOR in R. A filter of $>1.5$-fold change was set. Venn diagrams were generated by VENNY v.2.1.0 (http://bioinfogp.cnb.csic.es/tools/venny/index.html). $\mathrm{MeV}$ (http://mev.tm4.org/\#/welcome) was used to generate a heatmap and perform $\mathrm{k}$-mean clustering. A gene ontology (GO) term enrichment analysis was conducted using the agriGO web-based tool and database ${ }^{35}$ (http://bioinfo.cau.edu.cn/ agriGO/). The TreeMap view and interactive graph view of GO terms were generated with REVIGO (http://revigo.irb.hr/) after minimizing redundantly enriched GO terms ${ }^{68}$

Mutant phenotyping. To minimize environmental differences in growth chambers, the plants used for phenotyping were grown side-by-side at the same density in pots. The first five fruits from six different plants were observed for each genotype. A Chi-Square test was conducted to evaluate the statistical significance of the observed data.

Chemical treatment. For dex treatment, the inflorescence containing $g C R C-G R$, $35 S:: A G-G R$, or $35 S:: A P 1-G R$ were sprayed once with $10 \mu \mathrm{M}$ dex and $0.015 \%$ Silwet when plant heights were $4-8 \mathrm{~cm}$. The same amount of solvent and Silwet was used as a control. For expression analysis, RNA from $c r c-1$ gCRC-GR, ag-1 35S::AG-GR, Ler, $g C R C-G R$, 35S::AG-GR, and $g C R C-G R$ 35S::AG-GR was extracted four hours after dex treatment. For ChIP, ap1 cal-1 35S::AP1-GR gCRC-myc was fixed for four days after dex treatment when most floral buds were in developmental stage 6. For auxin treatment, a $30 \mu \mathrm{L}$ solution of $10 \mu \mathrm{M}$ NAA (Wako) and $0.015 \%$ Silwet was dropped onto inflorescences. Seven days after treatment, phenotype was observed.

Plasmid construction and plant transformation. For $g C R C-G F P$ construction, a genomic fragment covering 3492-bp upstream of the CRC translation start site and 1204-bp of the coding region was amplified from genomic DNA extracted from Ler and cloned into the binary vector pGreen0311 (for mGFP). To construct $p Y U C 4:$ : GUS, the $3.7 \mathrm{kbp}$ YUC4 promoter was amplified using genomic DNA from Ler as the PCR template and sub-cloned into pENTR/D-TOPO (Thermo Fisher Scientific) according to the manufacturer's protocol (Thermo Fisher Scientific). Site-directed mutagenesis was conducted using a PrimeSTAR Mutagenesis Basal Kit (Takara) to obtain $p Y U C 4 m$-pENTR/D-TOPO. The resulting $p Y U C 4$ fragments were Gateway-cloned into $p B G W F S 7$ with the LR reaction. To construct $p C H R 11::$ GUS and $p C H R 17:: G U S$, the $1 \mathrm{kbp} C H R 11$ promoter and $0.5 \mathrm{kbp}$ CHR17 promoter were amplified using genomic DNA from Col as the PCR template and sub-cloned into pENTR/D-TOPO according to manufacturer's protocol (Thermo Fisher Scientific). The resulting $p C H R 11$ and $p C H R 17$ fragments were Gateway-cloned into $p B G W F S 7$ vector by the LR reaction. To generate $35 S:$ GFP-CHR11, the 3.1 kbp CHR11 cDNA was amplified using cDNA synthesized from Col as the PCR template and was sub-cloned into pENTR/D-TOPO according to the manufacturer's protocol (Thermo Fisher Scientific). The resulting CHR11 fragment was Gateway-cloned into the $p G W B 6$ vector by the LR reaction. To generate pCRC::YUC4, the $3.5 \mathrm{kbp} C R C$ promoter and YUC4 coding sequence were amplified using genomic DNA and cDNA from Ler as PCR templates and the products were sub-cloned into different entry vectors. Whole functional fragments were cloned into the $p B G W$ destination vector.

To generate deletion vectors, gRNAs fused with Arabidopsis tRNA-Gly gene were amplified using the artificial DNA fragment (gBlock Gene Fragments) as the PCR template (Supplementary Data 3; Integrated DNA Technology). The resulting PCR products harboring two independent gRNA sequences were cloned into the AarI-digested pKI1.1R vector ${ }^{69}$ using In-Fusion reaction according to the manufacturer's protocol (Clontech). All insertion sequences were confirmed with an ABI $3130 \times 1$ Sequencer $(\mathrm{ABI})$ and $3130 \times 1$ series Data Collection 4 software (ABI). All primers used for cloning and genotyping are listed in Supplementary Data 3.

For plant transformations, inflorescences were dipped in Agrobacterium tumefaciens containing plasmids for $1 \mathrm{~min}$ and incubated for 1 day in humid conditions at room temperature. T1 seeds were collected and screened for antibiotic resistance. More than $20 \mathrm{~T} 1$ plants were screened, and representative lines were chosen for further characterization.

qRT-PCR. Using an RNeasy Plant Mini Kit (Qiagen), total RNA was extracted from Arabidopsis floral bud clusters up to stage 7 and stage 10 for $Y U C 4 / A G / C R C$ and WUS expression analysis, respectively. Genomic DNA was digested using an RNase-free DNase Set (Qiagen) prior to cDNA synthesis. Reverse transcription was performed using the PrimeScript RT Master Mix (Takara) according to manufacturer's protocol. The concentration of cDNA was determined with a Light Cycler 480 (Roche) and the Light Cycler 480 release 1.5.1.62 SP software (Roche) using the FastStart DNA Essential DNA Green Master (Roche). The detected signal was normalized to that of the internal control gene, EIF4 (At3g13290). The qRT-PCR experiments were repeated three times with more than four technical replicates. The values are represented as the means \pm SEMs. Two-tailed Student's $t$-test was conducted to evaluate the statistical significance of the observed data. Primers used for qRT-PCR are listed in Supplementary Data 3.

Confocal microscopy. For confocal microscope observations, plants were sown on vermiculite and Metro-Mix until plant height was $2-4 \mathrm{~cm}$. The apical $0.5 \mathrm{~cm}$ of the inflorescence tips was removed with tweezers, immediately embedded into $5 \%$ agar (Difco), and sliced with a Liner Slicer PRO7 vibratome (Dosaka). The resulting plant sections were placed on glass slides, mounted on a drop of water, and immediately observed under a FV 1000 (Leica) microscope with FV10-ASW software (Leica). More than 10 plants were observed, and representative images are shown.

Phylogenetic shadowing. The $5^{\prime}$ intergenic sequence of YUC4 from Arabidopsis thaliana was downloaded from TAIR. The YUC4 promoter region was used as a query to obtain the YUC4 promoter sequence in the other seven Brassicaceae species using NCBI blastn. The resulting eight YUC4 promoter sequences were aligned by mVISTA (http://genome.lbl.gov/vista/mvista/submit.shtml), and the promoter sequences were aligned using CLUSTALW (http://www.genome.jp/tools/ clustalw/). Conserved cis-elements were visualized by WebLogo (http://weblogo. berkeley.edu).

ChIP. For the CRC ChIP in the ap $1 \mathrm{cal}$ double mutant background, $0.3 \mathrm{~g}$ of inflorescences was used. The ap1-1 cal-1 35S::AP1-GR gCRC-myc inflorescences were treated once with $10 \mu \mathrm{M}$ DEX and harvested at 4 days after treatment, which correspond to stage 6-7. For the Pol II, AG, and CHR11-GFP ChIPs, $0.6 \mathrm{~g}$ of inflorescences was used. Inflorescences were fixed in $1 \%$ formaldehyde for fifteen minutes. To extract chromatin, fixed frozen tissues were homogenized with a mortar and pestle. The resulting chromatin was sonicated to produce DNA fragments shorter than $500 \mathrm{bp}$ and washed twice. IP was performed overnight. The myc (9E10; Santa Cruz), Pol II (4E8; Abcam), AG ${ }^{70}$, and GFP (A6455; Thermo Fisher Scientific) antibodies were used. After a wash with low and high salt buffer $^{71}$, reverse cross-linking was conducted. The resulting DNA was purified using a Qiaquick DNA Purification Kit (Qiagen). DNA was quantified with a Light Cycler 480 (Roche) and Light Cycler 480 release 1.5.1.62 SP software (Roche) using 
FastStart DNA Essential DNA Green Master (Roche). The ChIP experiments were repeated three times with more than three technical replicates. The values are represented as the means \pm SEMs. Two-tailed Student's $t$-test was conducted to evaluate the statistical significance of the observed data. TA3 was used as the internal control. Primers used in the ChIP experiment are listed in Supplementary Data 3.

Scanning electron microscopy. Fruits were fixed in FAA overnight at room temperature and dehydrated with an ethanol and acetone series. Critical point drying with liquid $\mathrm{CO}_{2}$ and a gold coating were performed using EM CPD300 (Leica) and E-1010 (Hitachi), respectively. The tissues were observed under an S4700 microscope (Hitachi) with an accelerating voltage of $15 \mathrm{kV}$. More than 10 fruits were observed under SEM, and representative images are shown.

FAIRE. For FAIRE, $0.3 \mathrm{~g}$ of inflorescences was fixed in $1 \%$ formaldehyde for eight minutes. To extract chromatin, fixed frozen tissues were homogenized with a mortar and pestle. The resulting chromatin was sonicated to produce DNA fragments shorter than $500 \mathrm{bp}$ and washed five times with wash buffer. To isolate nucleosome-depleted regions, a phenol-chloroform DNA extraction was performed ${ }^{61,72}$. After purification of DNA by Qiaquick DNA purification, DNA was quantified with a Light Cycler 480 (Roche) and the Light Cycler 480 release 1.5.1.62 SP software (Roche) using FastStart DNA essential DNA Green Master (Roche). FAIRE experiments were repeated twice with four technical replicates and the combined data are shown. Two-tailed Student's $t$-test was conducted to evaluate the statistical significance of the observed data. Primers used for FAIRE are listed in Supplementary Data 3.

Yeast two-hybrid assay. The AG, CRC, ARF19, and IAA14 cDNA fragments were amplified using PrimeSTAR GXL DNA Polymerase (Takara). Complementary DNA was prepared from RNA extracted from Arabidopsis Col and used as the PCR template. The resulting DNA fragments were introduced into pENTR/D-TOPO according to the manufacturer's protocol (Thermo Fisher Scientific). Then, cDNA fragments were Gateway-cloned into the $p D E S T$ vector (Thermo Fisher Scientific) by the LR reaction. Bait and pray constructs were co-transformed into the MaV203 yeast strain. Selection for double transformants was performed on -Trp-Leu SD media. A survival test was conducted using -Trp-Leu-His SD media. Empty constructs and ARF19 and IAA14 interaction were examined as negative and positive controls, respectively ${ }^{73}$.

Auxin measurement. For auxin measurements, floral buds were harvested when plant height reached $4-8 \mathrm{~cm}$. Flowers older than stage 8 were manually removed using a dissecting microscope. One-hundred milligrams of floral bud clusters was used. Auxin was extracted and semi-purified ${ }^{74}$. IAA was quantified with an ultrahigh performance liquid chromatography (UHPLC)-electrospray interface (ESI) and quadrupole-orbitrap mass spectrometer (UHPLC/Q-Exactive ${ }^{\mathrm{mx}}$; Thermo Fisher Scientific) with an ODS column ${ }^{75}$ (AQUITY UPLC HSS T3, $1.8 \mu \mathrm{m}, 2.1 \times 100 \mathrm{~mm}$; Waters). Auxin measurements were repeated twice, and the combined data were shown.

Transmission electron microscopy. For TEM, inflorescences were harvested when plants were $4-8 \mathrm{~cm}$ tall. Tissues were fixed in $2 \%$ paraformaldehyde and $1.25 \%$ glutaraldehyde in $0.05 \mathrm{M} \mathrm{PB}$ buffer for five hours at $4^{\circ} \mathrm{C}$, washed five times with $0.05 \mathrm{M}$ PB buffer for $10 \mathrm{~min}$ at $4{ }^{\circ} \mathrm{C}$, and fixed with $\mathrm{OsO}_{4}$ buffer at $4{ }^{\circ} \mathrm{C}$ overnight. The resulting tissues were washed with $8 \%$ sucrose in water for two hours at $4{ }^{\circ} \mathrm{C}$, dehydrated with an ethanol series, and infiltrated with Eponate 812 by incubating the samples at room temperature for several hours to overnight in increasing concentrations of resin. Then, the resin was polymerized in an oven at $60^{\circ} \mathrm{C}$ for $48 \mathrm{~h}$. Resin-embedded samples were sectioned to $70 \mathrm{~nm}$ widths with a diamond knife on a ultramicrotome. Sections were collected on a $0.5 \%$ formvar coated slot grid. Grids were post-stained for $5 \mathrm{~min}$ with $2 \%$ aqueous uranyl acetate and for 10 min with Reynolds lead citrate. Images were taken using a H-7100 TEM (Hitachi).

Sectioning. Fruits were fixed in FAA overnight at room temperature, dehydrated with an ethanol series, embedded in Technovit 7100 resin (Heraeus), and polymerized at room temperature overnight. Sections were made with a RM2255 microtome (Leica), extended onto slide glasses with a drop of water, and dried on a hot plate at $40^{\circ} \mathrm{C}$. After background staining with toluidine blue, sections were observed under an Axio Scope A1 microscope (ZEISS) equipped with an AxioCam ERc $5 \mathrm{~s}$ camera (ZEISS) and analyzed using ZEN2 software (ZEISS). More than five sections were observed, and representative images were shown.

GUS staining. Inflorescences of $4-8 \mathrm{~cm}$ in height were fixed in acetone for $20 \mathrm{~min}$ at room temperature, rinsed with water, and stained with GUS staining solution. Sectioning was performed as stated above starting with dehydration in $70 \%$ ethanol through Technovit sectioning. For background staining, $0.05 \%$ neutral red was used. Sections were observed under an Axio Scope Al microscope (ZEISS) equipped with an AxioCam ERc $5 \mathrm{~s}$ camera (ZEISS) and analyzed using the
ZEN2 software (ZEISS). To avoid positional effects of the transgene, exactly the same line was used in the wild type and mutants, generated by crossing. More than five sections were observed, and representative images are shown.

Immunofluorescence localization. Sectioning was performed as described in the sectioning section. For fixation, cacodylic acid was used instead of $\mathrm{FAA}^{76}$. All monoclonal antibodies (JIM7, LM5, and LM15) were obtained from Plantprobes (http://www.plantprobes.net/index.php) and used at a dilution of 1/10 (v/v) as primary antibodies. 2/10 (v/v) was used for Supplementary Fig. 16f and g. Alexa Fluor 488 (Thermo Fisher Scientific) was used for labeling. Immunolocalization experiments were performed at least three times per treatment using three independent sections, and representative images are shown.

In situ hybridization. Inflorescences of $4-8 \mathrm{~cm}$ in height were fixed in FAA at room temperature, dehydrated in a graded ethanol series, replaced with xylene and embedded in Paraplast (Sigma). Then, $8 \mu \mathrm{m}$ Palaffin sections were made using a RM2255 microtome (Leica). Sections were dewaxed and rehydrated. Hybridization was conducted in a humid chamber at $55^{\circ} \mathrm{C}$ overnight. The resulting sections were washed with SSC. Antibody binding and detection were performed using a DIG Labeling Kit (Roche). The 907-bp YUC4 probe was used to detect YUC4 mRNA. Primers used in the in situ hybridization are listed in Supplementary Data 3.

\section{Data availability}

Data supporting the findings of this study are available from the corresponding author upon reasonable request. A reporting summary for this Article is available as a Supplementary Information file.

Received: 14 June 2018 Accepted: 20 November 2018

Published online: 11 December 2018

\section{References}

1. O'Maoileidigh, D. S., Graciet, E. \& Wellmer, F. Gene networks controlling Arabidopsis thaliana flower development. New Phytol. 201, 16-30 (2014).

2. Sun, B. \& Ito, T. Regulation of floral stem cell termination in. Arab. Front Plant Sci. 6, 17 (2015)

3. Yanofsky, M. F. et al. The protein encoded by the Arabidopsis homeotic gene agamous resembles transcription factors. Nature 346, 35-39 (1990).

4. Bowman, J. L., Smyth, D. R. \& Meyerowitz, E. M. Genetic interactions among floral homeotic genes of Arabidopsis. Development 112, 1-20 (1991).

5. Mizukami, Y. \& Ma, H. Ectopic expression of the floral homeotic gene AGAMOUS in transgenic Arabidopsis plants alters floral organ identity. Cell 71, 119-131 (1992).

6. Huang, Z. et al. APETALA2 antagonizes the transcriptional activity of AGAMOUS in regulating floral stem cells in Arabidopsis thaliana. New Phytol. 215, 1197-1209 (2017).

7. Laux, T., Mayer, K. F., Berger, J. \& Jurgens, G. The WUSCHEL gene is required for shoot and floral meristem integrity in Arabidopsis. Development 122, 87-96 (1996)

8. Liu, X. et al. AGAMOUS terminates floral stem cell maintenance in Arabidopsis by directly repressing WUSCHEL through recruitment of Polycomb Group proteins. Plant Cell 23, 3654-3670 (2011).

9. Payne, T., Johnson, S. D. \& Koltunow, A. M. KNUCKLES (KNU) encodes a $\mathrm{C} 2 \mathrm{H} 2$ zinc-finger protein that regulates development of basal pattern elements of the Arabidopsis gynoecium. Development 131, 3737-3749 (2004).

10. Sun, B., Xu, Y., Ng, K. H. \& Ito, T. A timing mechanism for stem cell maintenance and differentiation in the Arabidopsis floral meristem. Genes Dev. 23, 1791-1804 (2009).

11. Sun, B. et al. Timing mechanism dependent on cell division is invoked by Polycomb eviction in plant stem cells. Science 343, 1248559 (2014).

12. Alvarez, J. \& Smyth, D. R. CRABS CLAW and SPATULA, two Arabidopsis genes that control carpel development in parallel with AGAMOUS. Development 126, 2377-2386 (1999).

13. Bowman, J. L. \& Smyth, D. R. CRABS CLAW, a gene that regulates carpel and nectary development in Arabidopsis, encodes a novel protein with zinc finger and helix-loop-helix domains. Development 126, 2387-2396 (1999).

14. Gomez-Mena, C., de Folter, S., Costa, M. M., Angenent, G. C. \& Sablowski, R Transcriptional program controlled by the floral homeotic gene AGAMOUS during early organogenesis. Development 132, 429-438 (2005).

15. Lee, J. Y. et al. Activation of CRABS CLAW in the nectaries and carpels of Arabidopsis. Plant Cell 17, 25-36 (2005)

16. Prunet, N. et al. REBELOTE, SQUINT, and ULTRAPETALA1 function redundantly in the temporal regulation of floral meristem termination in Arabidopsis thaliana. Plant Cell 20, 901-919 (2008). 
17. Yamaguchi, N., Huang, J., Xu, Y., Tanoi, K. \& Ito, T. Fine-tuning of auxin homeostasis governs the transition from floral stem cell maintenance to gynoecium formation. Nat. Commun. 8, 1125 (2017).

18. Goodrich, J. et al. Gene regulates homeotic gene expression in Arabidopsis. Nature 386, 44-51 (1997).

19. Forderer, A., Zhou, Y. \& Turck, F. The age of multiplexity: recruitment and interactions of Polycomb complexes in plants. Curr. Opin. Plant Biol. 29, 169-178 (2016).

20. Cnops, G. et al. The TORNADO1 and TORNADO2 genes function in several patterning processes during early leaf development in Arabidopsis thaliana. Plant Cell 18, 852-866 (2006).

21. Chiu, W. H., Chandler, J., Cnops, G., Van Lijsebettens, M. \& Werr, W. Mutations in the TORNADO2 gene affect cellular decisions in the peripheral zone of the shoot apical meristem of Arabidopsis thaliana. Plant Mol. Biol. 63, 731-744 (2007).

22. Staldal, V., Sohlberg, J. J., Eklund, D. M., Ljung, K. \& Sundberg, E. Auxin can act independently of CRC, LUG, SEU, SPT, STY1 in style development but not apical-basal patterning of the Arabidopsis gynoecium. New Phytol. 180, 798-808 (2008).

23. Zhao, Y. Auxin biosynthesis. Arab. Book 12, e0173 (2014).

24. Stepanova, A. N. et al. TAA-mediated auxin biosynthesis is essential for hormone crosstalk and plant development. Cell 133, 177-191 (2008).

25. Cheng, Y., Dai, X. \& Zhao, Y. Auxin biosynthesis by the YUCCA flavin monooxygenases controls the formation of floral organs and vascular tissues in Arabidopsis. Genes Dev. 20, 1790-1799 (2006).

26. Weijers, D. \& Wagner, D. Transcriptional responses to the auxin hormone. Annu. Rev. Plant Biol. 67, 539-574 (2016).

27. Reinhardt, D., Mandel, T. \& Kuhlemeier, C. Auxin regulates the initiation and radial position of plant lateral organs. Plant Cell 12, 507-518 (2000).

28. Powell, A. E. \& Lenhard, M. Control of organ size in plants. Curr. Biol. 22, R360-R367 (2012).

29. Hamant, O. et al. Developmental patterning by mechanical signals in Arabidopsis. Science 322, 1650-1655 (2008).

30. Yang, W. et al. Regulation of meristem morphogenesis by cell wall synthases in Arabidopsis. Curr. Biol. 26, 1404-1415 (2016).

31. Perrot-Rechenmann, C. Cellular responses to auxin: division versus expansion. Cold Spring Harb. Perspect. Biol. 2, a001446 (2010).

32. Cosgrove, D. J. Loosening of plant cell walls by expansins. Nature 407, 321-326 (2000).

33. Rose, J. K., Braam, J., Fry, S. C. \& Nishitani, K. The XTH family of enzymes involved in xyloglucan endotransglucosylation and endohydrolysis: current perspectives and a new unifying nomenclature. Plant Cell Physiol. 43, 1421-1435 (2002).

34. Schmid, M. et al. A gene expression map of Arabidopsis thaliana development. Nat. Genet. 37, 501-506 (2005).

35. Du, Z., Zhou, X., Ling, Y., Zhang, Z. \& Su, Z. agriGO: a GO analysis toolkit for the agricultural community. Nucl. Acids Res. 38, W64-W70 (2010).

36. Ud-Din, A. et al. Inamullah, Efficient use of artificial micro-RNA to downregulate the expression of genes at the post-transcriptional level in Arabidopsis thaliana. Genet. Mol. Res. 15, 15027439 (2016).

37. Hothorn, M., Van den Ende, W., Lammens, W., Rybin, V. \& Scheffzek, K. Structural insights into the $\mathrm{pH}$-controlled targeting of plant cell-wall invertase by a specific inhibitor protein. Proc. Natl Acad. Sci. USA 107, 17427-17432 (2010).

38. Friedrichsen, D. M. et al. Three redundant brassinosteroid early response genes encode putative bHLH transcription factors required for normal growth. Genetics 162, 1445-1456 (2002).

39. Kiba, T. et al. The type-A response regulator, ARR15, acts as a negative regulator in the cytokinin-mediated signal transduction in Arabidopsis thaliana. Plant Cell Physiol. 44, 868-874 (2003).

40. OMaoileidigh, D. S. et al. Control of reproductive floral organ identity specification in Arabidopsis by the C function regulator AGAMOUS Plant Cell 25, 2482-2503 (2013).

41. Enders, T. A., Frick, E. M. \& Strader, L. C. An Arabidopsis kinase cascade influences auxin-responsive cell expansion. Plant J. 92, 68-81 (2017).

42. Steindler, C. et al. Shade avoidance responses are mediated by the ATHB-2 HD-zip protein, a negative regulator of gene expression. Development 126, 4235-4245 (1999).

43. Crawford, B. C. \& Yanofsky, M. F. HALF FILLED promotes reproductive tract development and fertilization efficiency in Arabidopsis thaliana. Development 138, 2999-3009 (2011)

44. Colombo, M. et al. A new role for the SHATTERPROOF genes during Arabidopsis gynoecium development. Dev. Biol. 337, 294-302 (2010).

45. Jung, J. H., Lee, S., Yun, J., Lee, M. \& Park, C. M. The miR172 target TOE3 represses AGAMOUS expression during Arabidopsis floral patterning. Plant Sci. 215-216, 29-38 (2014)
46. Gan, Y. et al. GLABROUS INFLORESCENCE STEMS modulates the regulation by gibberellins of epidermal differentiation and shoot maturation in Arabidopsis. Plant Cell 18, 1383-1395 (2006).

47. Muller, C. J., Larsson, E., Spichal, L. \& Sundberg, E. Cytokinin-auxin crosstalk in the gynoecial promodium ensures correct domain patterning. Plant Physiol. 175, 1144-1157 (2017).

48. Ito, T. et al. The homeotic protein AGAMOUS controls microsporogenesis by regulation of SPOROCYTELESS. Nature 430, 356-360 (2004).

49. Zhang, W., Zhang, T., Wu, Y. \& Jiang, J. Genome-wide identification of regulatory DNA elements and protein-binding footprints using signatures of open chromatin in Arabidopsis. Plant Cell 24, 2719-2731 (2012).

50. Shamimuzzaman, M. \& Vodkin, L. Genome-wide identification of binding sites for NAC and YABBY transcription factors and co-regulated genes during soybean seedling development by ChIP-Seq and RNA-Seq. BMC Genom. 14, 477 (2013).

51. Yu, H., Ito, T., Wellmer, F. \& Meyerowitz, E. M. Repression of AGAMOUSLIKE 24 is a crucial step in promoting flower development. Nat. Genet. 36, 157-161 (2004)

52. Fletcher, J. C., Brand, U., Running, M. P., Simon, R. \& Meyerowitz, E. M. Signaling of cell fate decisions by CLAVATA3 in Arabidopsis shoot meristems. Science 283, 1911-1914 (1999).

53. Weigel, D., Alvarez, J., Smyth, D. R., Yanofsky, M. F. \& Meyerowitz, E. M. LEAFY controls floral meristem identity in Arabidopsis. Cell 69, 843-859 (1992).

54. Ehlting, J. et al. Global transcript profiling of primary stems from Arabidopsis thaliana identifies candidate genes for missing links in lignin biosynthesis and transcriptional regulators of fiber differentiation. Plant J. 42, 618-640 (2005).

55. Huanca-Mamani, W., Garcia-Aguilar, M., Leon-Martinez, G., Grossniklaus, U. \& Vielle-Calzada, J. P. CHR11, a chromatin-remodeling factor essential for nuclear proliferation during female gametogenesis in Arabidopsis thaliana. Proc. Natl Acad. Sci. USA 102, 17231-17236 (2005).

56. $\mathrm{Li}, \mathrm{G}$. et al. Imitation Switch chromatin remodeling factors and their interacting RINGLET proteins act together in controlling the plant vegetative phase in Arabidopsis. Plant J. 72, 261-270 (2012).

57. $\mathrm{Li}, \mathrm{G}$. et al. ISWI proteins participate in the genome-wide nucleosome distribution in Arabidopsis. Plant J. 78, 706-714 (2014).

58. Smaczniak, C. et al. Characterization of MADS-domain transcription factor complexes in Arabidopsis flower development. Proc. Natl Acad. Sci. USA 109, 1560-1565 (2012).

59. Yen, K., Vinayachandran, V., Batta, K., Koerber, R. T. \& Pugh, B. F. Genomewide nucleosome specificity and directionality of chromatin remodelers. Cell 149, 1461-1473 (2012).

60. Zhang, T., Zhang, W. \& Jiang, J. Genome-wide nucleosome occupancy and positioning and their impact on gene expression and evolution in plants. Plant Physiol. 168, 1406-1416 (2015).

61. Omidbakhshfard, M. A., Winck, F. V., Arvidsson, S., Riano-Pachon, D. M. \& Muller-Roeber, B. A step-by-step protocol for formaldehyde-assisted isolation of regulatory elements from Arabidopsis thaliana. J. Integr. Plant Biol. 56, 527-538 (2014).

62. Wagner, D., Sablowski, R. W. \& Meyerowitz, E. M. Transcriptional activation of APETALA1 by LEAFY. Science 285, 582-584 (1999).

63. Reyes-Olalde, J. I. et al. The bHLH transcription factor SPATULA enables cytokinin signaling, and both active auxin biosynthesis and transport genes at the medial domain of the gynoecium. PLoS Genet. 13, e1006726 (2017).

64. $\mathrm{Xu}, \mathrm{Y}$. et al. SUPERMAN regulates floral whorl boundaries through control of auxin biosynthesis. EMBO J. 37, e97499 (2018).

65. Clapier, C. R. \& Cairns, B. R. The biology of chromatin remodeling complexes. Annu. Rev. Biochem. 78, 273-304 (2009).

66. Uemura, A. et al. Regulation of floral meristem activity through the interaction of AGAMOUS, SUPERMAN, and CLAVATA3 in Arabidopsis. Plant Reprod. 31, 89-105 (2018).

67. Lin, D., Lopez-Sanchez, P. \& Gidley, M. J. Interaction of pectins with cellulose during its synthesis in the absence of calcium. Food Hydrocoll. 52, 57-68 (2016).

68. Supek, F., Bosnjak, M., Skunca, N. \& Smuc, T. REVIGO summarizes and visualizes long lists of gene ontology terms. PLoS ONE 6, e21800 (2011).

69. Tsutsui, H. \& Higashiyama, T. pKAMA-ITACHI vectors for highly efficient CRISPR/Cas9-mediated gene knockout in Arabidopsis thaliana. Plant Cell Physiol. 58, 46-56 (2017).

70. Ito, T., Takahashi, N., Shimura, Y., Okada, K. \& Serin, A. Threonine protein kinase gene isolated by an in vivo binding procedure using the Arabidopsis floral homeotic gene product, AGAMOUS. Plant Cell Physiol. 38, 248-258 (1997).

71. Yamaguchi, N. et al. Chromatin immunoprecipitation from Arabidopsis tissues. Arab. Book 12, e0170 (2014).

72. Wu, M. F. et al. Auxin-regulated chromatin switch directs acquisition of flower primordium founder fate. e-Life 4, e09269 (2015). 
73. Fukaki, H., Nakao, Y., Okushima, Y., Theologis, A. \& Tasaka, M. Tissuespecific expression of stabilized SOLITARY-ROOT/IAA14 alters lateral root development in Arabidopsis. Plant J. 44, 382-395 (2005).

74. Kojima, M. et al. Highly sensitive and high-throughput analysis of plant hormones using MS-probe modification and liquid chromatography-tandem mass spectrometry: an application for hormone profiling in Oryza sativa. Plant Cell Physiol. 50, 1201-1214 (2009).

75. Shinozaki, Y. et al. Ethylene suppresses tomato (Solanum lycopersicum) fruit set through modification of gibberellin metabolism. Plant J. 83, 237-251 (2015).

76. Freshour, G. et al. Developmental and tissue-specific structural alterations of the cell-wall polysaccharides of Arabidopsis thaliana roots. Plant Physiol. 110, 1413-1429 (1996).

\section{Acknowledgements}

We would like to thank Akie Takahashi, Hitomi Ichiwaka, Kyoko Sunuma, Mayumi Nara, and Taeko Kawakami for technical assistance; and Makoto Shirakawa and Sachi Ando for checking the draft of this manuscript. This work was supported by a grant from the Japan Science and Technology Agency 'Precursory Research for Embryonic Science and Technology' (JPMJPR15QA), JSPS KAKENHI Grant-in-Aid for Scientific Research on Innovative Areas (Nos. 16H01468, 18H04782), JSPS KAKENHI Grant-in-Aid for Scientific Research B (No. 18H02465), the NAIST foundation, Takeda Science foundation, and the Sumitomo Foundation to N.Y., Japan Science and Technology Agency 'Precursory Research for Embryonic Science and Technology' (JPMJPR15Q6), and JSPS KAKENHI Grant-in-Aid for Early-Career Scientists (No. 18K14678) to S.S.S., JSPS KAKENHI Grant-in-Aid for Scientific Research on Innovative Areas (Nos. 16H01468, 18H04793) to T.K., JSPS KAKENHI Grant-in-Aid for Scientific Research C (No. 16K07389) to R.Y., JSPS KAKENHI Grant-in-Aid for Scientific Research on Innovative Areas (No. 24114001), and JSPS KAKENHI Grant-in-Aid for Scientific Research B and C (Nos. 16K07389, 17H03691) to K.N., JSPS KAKENHI Grant-in-Aid for Scientific Research on Innovative Areas (No. 17H06473) to H.S., the Mitsubishi Foundation, JSPS KAKENHI Grant-in-Aid for Scientific Research on Innovative Areas (Nos. 15H01234, 15H01356, 17H05843, 18H04839), JSPS KAKENHI Grant-in-Aid for Scientific Research A (No. 15H02405), Grant-in-Aid for challenging Exploratory Research (No. 15K14549), Temasek Life Sciences Laboratory (TLL), and the National Research Foundation Singapore under its Competitive Research Programme (CRP Award NRFCRP001-108) to T.I.

\section{Author contributions}

N.Y., H.J., and T.I. conceived and designed the experiments. N.Y. conducted all the experiments unless otherwise specified. H.J. generated the $p C R C:: Y U C 4$ and $g C R C-G F P$ transgenic lines and performed the in situ hybridization. S.S.S. generated the CRISPR vectors. Y.T., M.K., Y.T., T.K., and H.S. quantified auxin levels. M. A. conducted Y2H. R.Y. and K.N. performed preliminary experiments for the immunolocalization of cell wall components.

\section{Additional information}

Supplementary Information accompanies this paper at https://doi.org/10.1038/s41467018-07763-0.

Competing interests: The authors declare no competing interests.

Reprints and permission information is available online at http://npg.nature.com/ reprintsandpermissions/

Publisher's note: Springer Nature remains neutral with regard to jurisdictional claims in published maps and institutional affiliations.

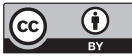

Open Access This article is licensed under a Creative Commons Attribution 4.0 International License, which permits use, sharing, adaptation, distribution and reproduction in any medium or format, as long as you give appropriate credit to the original author(s) and the source, provide a link to the Creative Commons license, and indicate if changes were made. The images or other third party material in this article are included in the article's Creative Commons license, unless indicated otherwise in a credit line to the material. If material is not included in the article's Creative Commons license and your intended use is not permitted by statutory regulation or exceeds the permitted use, you will need to obtain permission directly from the copyright holder. To view a copy of this license, visit http://creativecommons.org/ licenses/by/4.0/.

(C) The Author(s) 2018 